\title{
Réflexions sur l'appropriation française de l'analyse d'impact de la réglementation (A.I.R) dans le cas de la mise en œuvre des études d'impact des projets de loi
}

\section{> Patrick Gibert}

Professeur Émérite Université de Paris-Nanterre

\section{Résumé}

L'article débute par une présentation systématique des différentes finalités attribuées à l'analyse d'impact de la réglementation et un essai d'identification du degré de compatibilité ou d'antinomie entre la poursuite de ces différentes finalités. La seconde partie de l'article est un essai de caractérisation de l'appropriation de l'étude d'impact des projets de loi en France à la suite de l'obligation faite au gouvernement de présenter cette version de l'AIR pour la très grande majorité des projets de loi qu'il présente au parlement. Cet essai repose sur l'identification des paradigmes dominants dans la pratique française, sur le comportement des différentes parties prenantes aux études ainsi que sur une comparaison quantifiée des développements consacrés aux différents thèmes rendus obligatoires par la loi organique. La troisième partie replace les problèmes rencontrés par la pratique des études d'impact dans l'ensemble plus vaste de l'appropriation de la rationalité économico-managériale au sein du système politico-administratif français.

○ 2018 IDMP/Lavoisier SAS. Tous droits réservés

Mots clés : étude d'impact, légistique, évaluation ex ante, discipline du pouvoir..

\section{Abstract}

The ownership of the regulatory impact analysis, the French case. The article starts with a systematic presentation of the different purposes attributed to regulatory impact analysis and an attempt to identify the degree of compatibility or contradiction between the pursuit of these different purposes. The second part of the article is an attempt of characterization of the ownership of the impact study of the Bills in France as a result of the obligation of the Government to present this version of the RIA for most Bills submitted to the Parliament. This attempt is founded upon the identification of the dominant paradigms in the French practice, upon the behaviour of the different stakeholders in the studies as well as a quantified comparison of developments devoted to different themes required by the 2009 organic law. The

\footnotetext{
*Auteur correspondant : patrick.c.gibert@orange.fr

doi:10.3166/pmp.35. 2018.0012 @ 2018 IDMP/Lavoisier SAS - Tous droits réservés.
} 
third part replaces the problems faced by impact studies practice in the larger set of the ownership of economic and managerial rationality within the French political and administrative system.

(c) 2018 IDMP/Lavoisier SAS. Tous droits réservés

Keywords: regulatory impact analysis, legistics, ex ante evaluation, disciplining the decision-makers.

\section{Introduction}

Prônée par l'Organisation de Coopération et de développement économique (OCDE, 2008), pratiquée par l'Union Européenne et dans de nombreux États européens (Francesco, 2012), l'analyse d'impact de la réglementation (AIR) traduction française du « Regulatory Impact Assesment » (R.I.A) est un instrument de gestion ou d'action publique qui a déjà fait l'objet d'études et de bilans divers de la part très majoritairement de juristes mais aussi de politistes, sans que les spécialistes de sciences de gestion ne s'y soient véritablement attaqués. Pourtant son insertion délicate dans la « fabrique » des politiques publiques, tout comme les nombreux problèmes entraînés par sa mise en œuvre semble la rendre redevable d'une approche managériale.

L'AIR oblige à préciser les objectifs visés par un texte (loi ou règlement), à établir le lien entre les moyens utilisés et les effets attendus, à situer le dispositif proposé par rapport aux solutions alternatives. ${ }^{1}$ Autant d'activités qui évoquent, explicitement ou implicitement, la logique de l'analyse coût avantage (A.C.A) (Cost Benefit Analysis) et de l'évaluation a priori dont l'A.C.A a été et reste la variante privilégiée et la plus ambitieuse. Dans la doxa de l'administration fédérale américaine (Clinton, 1993 ; OMB, 2003 ; Obama, 2011; Trump, 2017) cette A.C.A tient la place centrale. ${ }^{2}$ Ce sont donc les concepts économiques qui prédominent dans la doctrine fédérale (OMB, 2003). Les échecs du marché dont l'existence d'externalités et de biens publics, le pouvoir de marché des entreprises, l'asymétrie d'information... sont évoqués comme les raisons de justification d'une intervention de l'État, avant l'énoncé d'autres fins sociales. Cependant l'AIR ne peut se résumer à l'ACA, la variété des façons dont elle est mise en œuvre (Radaelli, 2005) en témoigne.

En France l'analyse d'impact de la réglementation est dans le vocabulaire juridicoadministratif remplacée par la notion d'étude d'impact, en dépit de la polysémie de celle-ci.

L'expression étude d'impact est en effet utilisée pour désigner quatre dispositifs principaux. Le premier s'applique, en vertu d'une loi organique de 2009, à l'ensemble (ou presque Cf.

1 Pour I'O.C.D.E "RIA is a process of systematically identifying and assessing the expected effects of regulatory proposals, using a consistent analytical method, such as benefit/cost analysis. RIA is a comparative process: it is based on determining the underlying regulatory objectives sought and identifying all the policy interventions that are capable of achieving them. These "feasible alternatives" must all be assessed, using the same method, to inform decision-makers about the effectiveness and efficiency of different options and enable the most effective and efficient options to be systematically chosen." OCDE 2008 Pour I'Union Européenne "Impact assessment is about gathering and analysing evidence to support policy making. In this process, it verifies the existence of a problem, identifies its underlying causes, assesses whether EU action is needed, and analyses the advantages and disadvantages of available solutions". 2 Référence y est d'ailleurs faite au grand classique de Mishan EJ (1994), Cost-Benefit Analysis, fourth edition, Routledge, New York. 
infra) des projets de lois déposés par le gouvernement. Un deuxième, formalisé en dernier lieu par la circulaire du 17 juillet 2013, a trait aux textes réglementaires émanant du gouvernement et concerne «l'ensemble des textes applicables aux collectivités territoriales, aux entreprises ainsi qu'au public (particuliers, associations) ». Le troisième se rapporte à l'évaluation environnementale : «Les projets qui, par leur nature, leur dimension ou leur localisation, sont susceptibles d'avoir des incidences notables sur l'environnement ou la santé humaine font l'objet d'une évaluation environnementale $»^{3}$. Le quatrième, instauré par la loi du 7 août 2015 portant nouvelle organisation territoriale de la République, dite N.O.T.R.E, et précisé par un décret du 30 juin 2016, oblige les collectivités territoriales ainsi que leurs groupements à présenter à leur assemblée délibérante « Pour toute opération exceptionnelle d'investissement dont le montant est supérieur à un seuil fixé par décret en fonction de la catégorie et de la population de la collectivité ou de l'établissement... une étude relative à l'impact pluriannuel de cette opération sur les dépenses de fonctionnement » la même obligation s'applique pour les subventions d'investissement. Les seuils sont fixés par un décret du 30 juin 2016.

La même expression est donc utilisée pour trois types d'objets différents : des textes juridiques, des projets (pour faire court d'aménagement) qui s'inscrivent dans des territoires, des investissements de toute nature potentiellement générateurs de dépenses futures. Les études se rapportant au premier type d'objet peuvent s'assimiler à de l'analyse d'impact de la réglementation (au sens de régulation et non de normes générales et impersonnelles édictées par le pouvoir exécutif), les deuxième et troisième types en sont plus éloignés.

L'étude d'impact des projets de lois et celle des règlements (au sens juridique du terme cette fois) se différencient de plusieurs points de vue; elles relèvent d'abord d'un encastrement juridique extrêmement différent : une circulaire se situant en bas de la hiérarchie des normes pour l'étude d'impact des règlements, une loi organique qui ne le cède qu'à la Constitution pour l'étude d'impact des projets de loi; la justification de l'étude législative d'impact est, pour le moins, plurielle (Cf. infra), tandis qu'elle est très associée à un objectif affiché de simplification administrative pour l'étude d'impact des « règlements »; leur mode opératoire varie, d'un dossier qui peut atteindre des dizaines de pages pour les lois à une fiche assez schématique pour les règlements; elles se différencient enfin par l'audit de l'étude, en principe réalisé de façon systématique par le Conseil d'État dans le cas de l'étude législative d'impact, opéré par le Conseil national d'évaluation des normes et limité aux textes ayant des répercussions pour les collectivités territoriales en ce qui concerne l'étude des règlements. L'ensemble de ces considérations nous a amené à centrer notre étude sur le seul cas de l'étude d'impact législatif. Cette étude est régie par l'article 8 de la loi organique du 15 avril $2009 .{ }^{45}$

\footnotetext{
${ }^{3}$ De laquelle est distincte l'évaluation socio-économique obligatoire pour les grands projets d'infrastructure de transport. Pour une appréciation de la mise en œuvre de celle-ci Cf. autorité environnementale 2017.

${ }^{4}$ Marginalement modifié par la loi organique $n^{\circ} 2010-704$ du 28 juin 2010 relative au conseil économique et social ajoutant aux développements demandés « - s'il y a lieu, les suites données par le Gouvernement à l'avis du Conseil économique, social et environnemental; ».

${ }^{5}$ Les travaux à effectuer ont été augmentés par voie de circulaire de :

l'impact en termes d'égalité entre les hommes et les femmes (circulaire du 23 août 2012),

l'impact sur le handicap (circulaire du 4 septembre 2012),

l'impact sur la jeunesse (circulaire du 2 mai 2016 du premier ministre).
} 


\section{Encadré 1 : Dispositions de la loi organique du 15 avril 2009}

Article 8 Les projets de loi font l'objet d'une étude d'impact. Les documents rendant compte de cette étude d'impact sont joints aux projets de loi dès leur transmission au Conseil d'État. Ils sont déposés sur le bureau de la première assemblée saisie en même temps que les projets de loi auxquels ils se rapportent.

Ces documents définissent les objectifs poursuivis par le projet de loi, recensent les options possibles en dehors de l'intervention de règles de droit nouvelles et exposent les motifs du recours à une nouvelle législation. Ils exposent avec précision :

- l'articulation du projet de loi avec le droit européen en vigueur ou en cours d'élaboration, et son impact sur l'ordre juridique interne;

- l'état d'application du droit sur le territoire national dans le ou les domaines visés par le projet de loi;

- les modalités d'application dans le temps des dispositions envisagées, les textes législatifs et réglementaires à abroger et les mesures transitoires proposées;

- les conditions d'application des dispositions envisagées dans les collectivités régies par les articles 73 et 74 de la Constitution, en Nouvelle-Calédonie et dans les Terres australes et antarctiques françaises, en justifiant, le cas échéant, les adaptations proposées et l'absence d'application des dispositions à certaines de ces collectivités;

- l'évaluation des conséquences économiques, financières, sociales et environnementales, ainsi que des coûts et bénéfices financiers attendus des dispositions envisagées pour chaque catégorie d'administrations publiques et de personnes physiques et morales intéressées, en indiquant la méthode de calcul retenue;

- l'évaluation des conséquences des dispositions envisagées sur l'emploi public;

- les consultations qui ont été menées avant la saisine du Conseil d'État;

- la liste prévisionnelle des textes d'application nécessaires

Dans une première partie nous mettrons en exergue les différents paradigmes auxquels paraît se référer la pratique française de l'étude d'impact des projets de loi comme les critiques qui lui sont faites ou les voies d'amélioration suggérées par les milieux politico-administratifs.

Dans une seconde partie nous analyserons les études d'impact réalisées en 2016 et 2017 pour caractériser l'appropriation qui a été faite en France de l'analyse d'impact de la réglementation.

Dans une troisième partie nous avancerons l'idée que ce type d'appropriation n'est qu'une des manifestations de la façon dont sont utilisés en France depuis des lustres les instruments introduits par les dispositifs institutionnalisés de réforme de l'action publique.

\section{Le rattachement de I'AIR à de multiples paradigmes}

L'AIR est un instrument qui peut se rattacher à divers paradigmes. Par là même elle apparaît comme un objet frontière ${ }^{6}$ Cette caractéristique est à même d'expliquer à la fois

\footnotetext{
${ }^{6}$ L'objet frontière est " un concept analytique de ces objets scientifiques qui d'une part sont présents dans
} 
l'accord de principe quant à l'adoption de cet instrument et la variété des modes d'appropriations (Dunlop et al., 2012) par des utilisateurs qui privilégient, volontairement ou non, tel ou tel paradigme dans la façon dont ils s'approprient l'instrument. Il importe donc dans un premier temps de passer en revue les paradigmes sous-jacents à l'AIR, tels qu'ils apparaissent ou transparaissent dans les travaux ayant amené à la mise en œuvre des dispositifs d'étude d'impact, dans les analyses critiques concernant leur processus d'élaboration ou leur contenu ou encore dans les propositions de réforme dont les dispositifs ont fait l'objet.

Nous avons pu identifier dans la doxa politico-administrative dix paradigmes différents : celui de la mise en cause du pouvoir discrétionnaire, celui du rééquilibrage des pouvoirs, celui de la démocratie participative, le paradigme du libéralisme économique, celui de la simplification administrative, le paradigme de la lutte contre les lois bavardes, celui d'une bonne législation, le paradigme de la boucle évaluative, celui de la centralisation et du contrôle de l'action publique.

\subsection{Le paradigme de la mise en cause du pouvoir discrétionnaire}

L'obligation de l'AIR s'attaque frontalement au pouvoir discrétionnaire des gouvernants. Si celui-ci étymologiquement est fondé sur la capacité de discernement du décideur, il est en fait interprété comme la capacité de décider librement sous la seule condition du respect des normes d'ordre supérieur dans la hiérarchie de celles-ci (Constitutions et lois organiques dans le cas des lois « ordinaires ») et à l'exception (en tout cas en France) de l'erreur manifeste d'appréciation, hypothèse dans laquelle le juge sanctionne les cas trop flagrants d'abus de pouvoir. Cet état de fait n'a jamais empêché de développer la justification des textes, en particulier au moyen de l'exposé des motifs, mais cette justification, en l'absence d'A.I.R, peut se cantonner à une rhétorique (Devriendt et Monte, 2015) affirmant de façon plus ou moins péremptoire des liens entre les fins affichées et le dispositif d'un texte qui peut être en fait fondé sur une idéologie (entendue comme un système global de pensée), des valeurs, un système de pensée nullement réfutable (falsifiable) (Popper, 1957) plus ou moins conscients et explicités.

Le paradigme s'attaque de façon frontale au modèle de la poubelle avancé par Cohen, March et Olsen, à l'idée que la solution précède souvent les problèmes, que la formulation de ceux-ci est l'occasion de faire adopter les solutions qui constituent le véritable enjeu de la politique et que l'appariement des uns et des autres est un véritable bricolage (March et Olsen, 1989).

L'obligation d'une AIR paraît instaurer, au moins implicitement, une discipline du pouvoir (Gibert et Verrier, 2016) Elle substitue la logique de la démonstration à celle de l'affirmation, celle de l'explication analytique à celle des propositions générales plus ou moins fournies par des idéologies, des croyances, ou le sens commun (sans compter les mesures purement clientélistes destinées à donner satisfaction à une catégorie particulière d'électeurs). Elle met potentiellement sur le forum des mesures que les gouvernants auraient pu vouloir faire adopter discrètement. Les deux premières substitutions visent à promouvoir la rationalité managériale, la troisième à diminuer les effets pervers de la discrétion politique. Si l'on

plusieurs mondes sociaux s'entrecoupant (...) et qui satisfont les besoins d'information de chacun d'entre eux... » (Star et Griesemer, 1989) 
considère que cette démarche est caractéristique d'une rationalité managériale, alors que la traditionnelle légitimation des textes par la compétence juridique de l'autorité qui les édicte et le respect de la hiérarchie des normes est caractéristique de la rationalité juridique, on aboutit à l'idée que l'AIR est un élément de cette managérialisation du droit (Chevallier, 1993) annoncée depuis longtemps par les spécialistes de droit public. La réalité de cette managérialisation dépend du respect de normes un peu strictes d'élaboration des analyses (que l'on peut commodément sinon justement résumer par le terme de qualité des études) et, au-delà, de l'effectivité (efficacy) des A.I.R entendue comme leur capacité à produire des effets c'est-à-dire à modifier concrètement le comportement des décideurs que ce soit quant aux choix opérés, quant à la communication organisée autour de ces choix, quant au process de confection de la norme... selon les paradigmes au nom desquels dans un pays donné on instaure l'AIR.

Une des limites fondamentales du paradigme de la limitation du caractère discrétionnaire du pouvoir est qu'il privilégie de fait le caractère instrumental d'une loi qui est largement concurrencé dans l'agenda et le débat politiques par les aspects idéologiques et symboliques que chaque projet et la politique qu'il porte contiennent. La rationalité imposée implicitement au pouvoir par l'AIR renferme sans doute une opposition frontale avec une logique politique s'accommodant à l'inverse bien davantage du flou, de l'ambiguïté (Chun et Rainey, 2006) et de l'abstraction (Moor, 1995), dans l'expression des intentions ainsi que dans celle du lien entre les fins poursuivies et les moyens utilisés. Plus globalement la limitation du caractère discrétionnaire du pouvoir paraît en porte à faux avec la vision des analystes de politique, imprégnée d'une vision selon laquelle «Les problèmes politiques sont des construits sociaux reflétant des conceptions particulières de la réalité. Les choix effectués dans le processus de construction de l'agenda ont des conséquences potentielles importantes en ce sens qu'ils affectent les réalités matérielles des vies des personnes et qu'ils influencent leurs interprétations de ces réalités » (Elder et Cobb, 1984). Adopter le paradigme c'est également occulter, au moins en partie, l'idée selon laquelle «les stratégies d'argumentation (sont) destinées à rendre légitimes les revendications » (Muller, 2005) au sein de systèmes où s'exercent toujours des rapports de force.

\subsection{Le paradigme du rééquilibrage des pouvoirs ${ }^{7}$}

Globalement le vingtième siècle a été caractérisé par la montée du pouvoir exécutif au détriment du pouvoir législatif. Montée observée de longue date aux États-Unis, plus récemment en France où elle date de la constitution de la Ve République conçue par le général de Gaulle et ses collaborateurs comme une réponse aux excès du parlementarisme des deux républiques précédentes. Il est important de noter que la loi organique de 2009 est en fait un texte d'exécution de la loi constitutionnelle de 2008 pour laquelle le Premier ministre de l'époque avait souligné tous les pouvoirs nouveaux qu'elle transférait au Parlement (Denolle, 2011). Sous le paradigme du rééquilibrage des pouvoirs il

\footnotetext{
${ }^{7}$ Rééquilibrage ne signifie pas fin de la séparation des pouvoirs, le Conseil constitutionnel l'a rappelé en 2009 en censurant la proposition selon laquelle les projets de loi faisaient l'objet d'une étude d'impact " dès le début de leur élaboration » et dans le texte initial « la liste prévisionnelle des textes d'application nécessaires, leurs orientations principales et le délai prévisionnel de leur publication » les deux dernières propositions pour méconnaissance de la séparation des pouvoirs, (CC 2009).
} 
s'agit d'amener le pouvoir exécutif à justifier ses projets de loi autrement que dans les termes très généraux qui caractérisent depuis longtemps les exposés des motifs de ces dits projets. Le fait que la loi organique de 2009 n'impose les études d'impact que pour les projets, et pas pour les propositions de lois (qui émanent des parlementaires) ou pour les amendements déposés par les parlementaires paraît être un indice fort de la vigueur de ce paradigme dans le cas français ${ }^{8}$.

Pour Pascale Deumier (colloque AN, 2014 : 25) «L'étude d'impact est... un outil de rationalisation des rapports entre les pouvoirs exécutif et législatif : elle suscite un débat entre eux et permet d'établir un lien entre l'initiative prise par l'un et le contrôle exercé par l'autre »

On reproche souvent à ce paradigme de reposer sur une opposition purement juridique et de faire fi de réalités sociologiques, ordinairement fortes, à savoir que la véritable confrontation est celle de la majorité présidentielle et de l'opposition, gouvernement et majorité se retrouvant dans un même camp qui n'a pas forcément intérêt à l'explicitation de la logique d'un texte. Si l'on admet ce point de vue, l'étude d'impact menée dans les règles de l'art n'est donc pas un document traduisant la nécessité d'une pleine explicitation de sa logique par un pouvoir (l'exécutif) au profit de l'autre (le législatif) mais un document constituant une ressource (au sens de la sociologie des organisations) au profit de l'opposition qui peut en dénoncer tant le caractère contestable des chaînes causales mise en lumière que celui des quantifications éventuellement opérées ou encore l'absence des divers développements dont la loi organique rend la présence obligatoire, dès lors que défauts ou lacunes peuvent être sanctionnés par le juge, en l'occurrence constitutionnel. La première utilisation mentionnée de la ressource traduirait une appropriation réelle de la logique de la loi organique par les parlementaires, la seconde peut s'analyser comme une appropriation opportuniste dans la mesure où, pour éviter une loi à laquelle ils sont hostiles, les parlementaires appartenant à une opposition, quelle qu'elle soit, sont habitués à faire feu de tout bois.

\subsection{Le paradigme de la démocratie participative}

Dans les normes relatives à L'AIR de l'Union Européenne l'association la plus en amont possible des parties prenantes dans le processus d'élaboration de l'étude d'impact est préconisée, «Les parties prenantes doivent pouvoir donner leur avis sur la préfiguration d'une analyse d'impact qui décrit le problème, les questions annexes qui peuvent y être reliées, les objectifs, les options possibles ainsi qu'une première estimation des impacts pertinents de ces options » (Commission européenne, 2009). Cette association en début de processus est effectivement pratiquée (Cour des comptes européenne, 2010). Il s'agit là d'une modalité visant à éviter des études d'impact conçues de façon trop endogamique, des études d'impact hors sol. En France tout citoyen peut faire parvenir ses remarques sur une étude d'impact à l'assemblée devant laquelle le gouvernement a déposé en premier (généralement l'assemblée nationale) un projet de loi ainsi que les documents préparatoires exigés par la loi organique de 2009 dont l'étude d'impact fait partie. Cette possibilité semble peu utilisée, et l'utilisation semble en trouver peu d'écho (colloque AN, 2014). Dans la

\footnotetext{
${ }^{8}$ En revanche le fait que les amendements émanant du gouvernement ne soient pas soumis à la même formalité reste inexpliqué dans ce cadre.
} 
formule participative le risque d'une participation biaisée a rapidement été mis en avant, les groupes les mieux organisés souvent liés aux intérêts économiques les plus puissants étant bien davantage a même de participer aux études, et de mener eux-mêmes études et contre études susceptibles d'influencer de façon significative le résultat final que ne le sont des acteurs plus isolés.

\subsection{Le paradigme libéral}

Le paradigme libéral se manifeste dans l'AIR par l'obligation d'énoncer les solutions alternatives à la norme envisagées et les raisons pour lesquelles l'auteur de la norme a renoncé à l'ensemble de ces solutions. Aux États-Unis par exemple c'est l'expression de l'idée selon laquelle le command and control doit s'exercer en dernier recours par rapport aux autres modalités d'action publique (OMB, 2003). Il existe une « présomption contre la réglementation en matière économique » dans plusieurs types de domaines comme le contrôle des prix ou l'imposition de normes de qualité ${ }^{9}$ qui a comme conséquence d'imposer « à la lumière de la théorie économique et de l'expérience » « une charge de la preuve particulièrement exigeante » pour les réglementations devant y intervenir. De façon générale ce sont les solutions les plus libérales qui doivent être retenues par l'administration : « des normes de performance plutôt que des normes portant sur la façon de procéder ${ }^{10}$ », des approches orientées marché reposant sur des incitations économiques plutôt que sur des contrôles directs, des mesures informationnelles plutôt que de la réglementation (ibidem).

En d'autres termes parmi les instruments d'action publique ou de gouvernance, (Hood, 1986 ; Halpern, Lascoumes et Le Galès, 2014) la règlementation est conçue comme un pisaller par rapport à la communication-persuasion, aux incitations financières, à la contractualisation... On notera au passage que chacun de ces modes alternatifs demande des textes juridiques pour pouvoir être appliqué - dans la mesure où il nécessite la mobilisation de l'argent public ou l'édiction de normes d'action aux administrations chargées de sa mise en œuvre et que c'est bien la norme contraignante au sens le plus strict du terme qui est visée (mieux vaut un marché des droits à polluer que la fixation de limites infranchissables pour chaque acteur économique pollueur, mieux vaut l'instauration de primes à l'achat de véhicules non polluants que l'interdiction d'utiliser des véhicules très polluants...) Le caractère libéral du paradigme ne concerne donc pas à proprement parler le champ d'intervention de l'État - et n'est pas assimilable à la recherche d'un État minimum ou d'un « moins d'État » - mais a plutôt trait à son mode d'intervention.

Le guide de légistique, en France, contient des développements proches de la doxa américaine sur ce point ${ }^{11}$.

\footnotetext{
${ }^{9}$ Parmi eux le contrôle des prix dans des marchés concurrentiels, les quotas de production ou de ventes dans ces mêmes marchés les standards de qualité uniformes et obligatoires pour des biens et services quand le problème potentiel peut être traité de façon adéquate par des standards volontaires ou par la communication sur le problème aux acheteurs ou consommateurs...

${ }^{10}$ Engineering or design standards

11 L'étude d'impact doit recenser «les options possibles en dehors de l'intervention de règles de droit nouvelles».
} 


\subsection{Le paradigme de la simplification administrative}

Ce paradigme se présente de fait sous deux versions. La première a trait à la complexité du droit pour tout administré, la seconde se rapporte plus spécifiquement aux normes s'appliquant aux entreprises, aux coûts et à l'insécurité juridique que ces normes entraînent pour elles. Pour ce second volet, les politiques de simplification administrative se fondent sur l'idée selon laquelle le droit d'un État est devenu un élément essentiel de la compétitivité internationale, aussi bien en raison des coûts qu'il engendre pour les entreprises que de l'image qu'il donne de la facilité ou de la difficulté qu'il y aurait à entreprendre dans un pays donné.

Rissi et Sager (2013) soulignent le fait qu'en Suisse, au niveau de la confédération les analyses d'impact de la réglementation ont été introduites comme « des instruments de réduction de la charge administrative.

Le paradigme de la simplification s'opérationnalise dans certains pays par l'édiction de règles brutales comme l'obligation de supprimer 1 voire 2 ou 3 règles existantes pour la mise en œuvre d'une règle nouvelle. Ainsi en France une circulaire de 2013 (Premier Ministre, 2013) prévoit une règle « une norme créée, une norme supprimée ou allégée », et plus précisément que « tout projet de texte réglementaire nouveau créant des charges pour les collectivités territoriales, les entreprises ou le public ne pourra être adopté que s'il s'accompagne, à titre de « gage », d'une simplification équivalente ». Aux États-Unis il est énoncé en 2017 que pour chaque nouvelle réglementation on doit identifier au moins deux réglementations existantes à éliminer », (Trump, 2017). Ce sont là des dispositifs qui empruntent souvent davantage à la symbolique qu'à une réelle logique d'efficacité dans la mesure où les règles supprimées sont souvent des règles tombées en désuétude (donc peu gênantes pour les entreprises) et que l'unité de mesure «nombre de règles » est très médiocre ${ }^{12}$. Au-delà de cette utilisation abusive d'un indicateur ambigu le problème essentiel du paradigme de la simplification vient du fait que le nombre de règles édictées dans un État, loin d'être une variable indépendante, dépend des politiques en matière fiscale, environnementale, du droit du travail, de la concurrence et des positions dominantes, règles qui pour les pays européens découlent pour une partie importante de dispositifs nationaux d'application de directives de l'Union; Les états de surcroît se livrent pour beaucoup d'entre eux à une surtransposition de ces directives. Une véritable simplification administrative est d'abord affaire de modestie interventionniste. L'adjectif «administrative » est trompeur dès lors qu'il peut laisser croire à une maladie de l'administration désireuse de justifier son existence par une logorrhée textuelle - situation qui ne peut pas être cependant totalement exclue-. La complexité des règles est également liée à des politiques de « fine tuning » visant à éviter le développement d'effets d'aubaine, d'effets pervers, inévitablement lié à des règles trop simples. La simplification administrative ressemble beaucoup à une attaque de symptômes plutôt qu'à un travail sur la racine du mal dès lors qu'elle ne porte pas sur une réflexion portant sur les différentes technologies de l'action publique, sur les avantages et les inconvénients des différents instruments d'action publique, chose sur laquelle la doxa de l'AIR insiste généralement sous la forme de l'obligation d'expliciter le pourquoi du renoncement à des solutions alternatives.

\footnotetext{
12 Le remplacement de trois règles simples ou légères par une seule règle complexe mais lourde ne sera pas vécu comme une simplification par les entreprises concernées. Simplification renvoie à complexité tout autant qu'à nombre
} 


\subsection{Le paradigme de la lutte contre les lois bavardes}

Ce paradigme semble plus particulièrement important en France. Les juges constitutionnel et administratif ont uni leurs efforts pour lutter contre la tendance du législateur à produire des textes qui pour tout ou partie n'ont aucune portée juridique réelle afin d'affirmer des principes avec souvent l'intention au moins supposée de satisfaire ainsi une partie de la population c'est-à-dire de l'électorat. Il peut s'agir de lois dites mémorielles ${ }^{13}$ mais aussi bien de portions de lois de toute nature développant des paragraphes sans portée réelle. La Loi est considérée comme «bavarde, précaire, banalisée » fruit d'une gesticulation législative des pouvoirs publics, ${ }^{14}$ et ce constat semble partagé par des responsables parlementaires (Bartelone 2013). La dénonciation de l'utilisation de la loi comme moyen de communication politique par le président du Conseil Constitutionne ${ }^{15}$, des avis négatifs du conseil d'État, dans sa fonction de conseiller du Gouvernement ${ }^{16}$, la censure du Conseil Constitutionnel ont traduit l'allergie grandissante des juges à la loi bavarde. Pour ne pas prêter le flanc à une dénonciation en méconnaissance de la « recherche de sens », ou en refus illégitime de la reconnaissance du rôle symbolique que revêt l'action du pouvoir, en sus de l'instrumentalité de ses activités le Conseil d'État a établi depuis 25 ans une distinction entre le texte législatif lui-même qui ne saurait être bavard et l'ensemble de son environnement : travaux préparatoires et exposé des motifs en particulier qui suffisent selon lui à répondre à cette quête de sens et de symboles (Conseil d'État rapport public annuel 1991).

\subsection{Le paradigme de la redevabilité des gouvernants}

L'idée que les gouvernants doivent rendre à leur mandat des comptes n'est pas neuve, mais dans nombre de pays elle s'est de facto limitée pendant longtemps, pour ce qui est des procédures formalisées et périodiques, à la redevabilité financière. La montée de la gestion de la performance dans le secteur public, la revendication de résultats de la part des citoyens ont entraîné un développement de la redevabilité de résultats matérialisée par exemple par les projets et les rapports annuels de performance associés aux lois de finances de l'État en application de la loi organique relative aux lois de finances (LOLF) (Benzerafa et Gibert, 2016). Ce « rendre compte » s’inscrit dans un cadre organisationnel, le découpage entre programmes ayant été très influencé par le respect des structures organisationnelles et reprenant, sauf exceptions, l'intégralité de l'action étatique dans un sous domaine d'action

\footnotetext{
${ }^{13}$ Dont certaines ont cependant une portée juridique dès lors que la négation des principes qu'elles posent ou des faits qu'elles rapportent est interdite sous peine de sanctions pénales.

${ }^{14} \mathrm{R}$ Denoix de Saint-Marc, alors vice-président du Conseil d'État. Interview par le Journal du dimanche. Janvier 2001

15 Jean louis Debré- alors président du Conseil Constitutionnel- interviewé par Acteurs publics octobre 2014

${ }^{16}$ Pour un exemple récent: Cf. Avis du conseil d'État sur le projet de loi «Égalité et citoyenneté ». Le Conseil « a écarté le premier des deux alinéas introduits à l'article L. 6111-2 du code du travail, au motif que ces dispositions ne présentaient pas de caractère normatif en ce qu'elles se bornaient à affirmer le principe que les actions de lutte contre l'illettrisme et en faveur de l'apprentissage et de l'amélioration de la maîtrise de la langue française constituent une " priorité nationale » et doivent s'inscrire dans une approche globale qui doit concerner tous les acteurs publics, sans en déduire des règles spécifiques relevant du domaine de la loi. »
} 
déterminé, quelle que soient l'ancienneté des dispositifs qui enserrent cette action; on ne dispose donc pas là d'un « rendre compte » des politiques générées à un moment donné du temps par l'action gouvernementale (Gibert, 2002). Pour le « rendre compte » des résultats de ces politiques on doit s'en remettre à des évaluations ex post, mais seule l'évaluation ex ante assure une redevabilité des gouvernants sur leurs intentions, leur logique et les effets attendus des politiques qu'ils désirent mettre en œuvre, c'est en ce sens que l'AIR rejoint le mouvement de la redevabilité.

\subsection{Le paradigme de la boucle évaluative}

L'AIR Apparaît également comme une nouvelle tentative pour obtenir que les politiques publiques soient évaluables ex post. C'est l'espoir par exemple du rapporteur de la mission d'information sur la simplification législative Régis Juanico, (colloque AN, 2014). C'est même un principe énoncé dans le guide de légistique édicté par le secrétariat général du gouvernement (SGG) : « on s'attachera dans la mesure du possible à faire le départ entre un objectiffinal et plusieurs objectifs intermédiaires, à déterminer un ou plusieurs indicateurs qui permettront de mesurer, même indirectement, si ces objectifs ont été atteints et à présenter la nature du dispositif d'évaluation ex post à envisager ». Pour Didier Migaud (Premier président de la Cour des Comptes) également, rendre les politiques publiques évaluables ex post est une nécessité (colloque AN, 2014) ${ }^{17}$. De surcroît le couple évaluation a priori, évaluation ex post est vu comme un gage de renforcement de la qualité des études a priori (Lambert in ibidem)

La nécessité d'expliciter les objectifs et de les opérationnaliser, le souci parfois énoncé d'un idéaltype qui serait l'analyse coût avantage -laquelle demande une quantification non seulement des effets recherchés mais aussi, en principe du moins, des autres effets -sont de nature à fournir aux évaluateurs ex post les critères qui leur manquent le plus souvent pour réaliser une véritable évaluation des résultats de la politique qu'ils étudient. Elle devrait leur éviter de devoir se cantonner à une étude de la seule mise en œuvre de la politique en question ou alternativement de voir dénoncer les indicateurs de résultats qu'ils utilisent ou élaborent pour l'occasion comme arbitraires au regard des intentions du législateur, peu à même qu'ils seraient d'estimer sans biais ou sans réduction illégitime celles-ci. ${ }^{18}$

\footnotetext{
17 "Évaluer une réforme qui n'a pas été conçue dès l'origine dans la perspective d'être, un jour, évaluée, est une tâche lourde, difficile et souvent improductive. II faut identifier les objectifs visés, qui ne sont pas toujours clairement formulés, trouver les données quantitatives et qualitatives permettant de voir si ces objectifs sont atteints et inciter les administrations elles-mêmes à évaluer l'impact des règles dont elles ont proposé l'adoption. Cela représente un travail important qui n'aboutit pas toujours, faute de données disponibles. Autrement dit, si l'on se contente de renforcer l'évaluation ex post, la Cour sera à même de livrer un nombre réduit de travaux. Si, au contraire, le législateur et l'administration conçoivent des dispositifs de qualité, qui identifient des objectifs clairs et prévoient la collecte de données afin de vérifier dans quelle mesure ils sont atteints, la Cour sera en mesure de vous livrer des évaluations ex post plus utiles et plus nombreuses ».

${ }^{18}$ On notera que l'explicitation a priori d'objectifs relativement précis pour une politique ne garantit ni la capacité qu'il y aurait à résoudre le problème de l'imputation à cette politique des changements d'état des phénomènes sur lesquels elle est censée agir, ni même la disponibilité des informations pour nourrir l'évaluation quantifiée des résultats et en particulier de ses impacts recherchés ou non.
} 


\subsection{Le paradigme centralisateur (ou du contrôle)}

Les sources des textes législatifs ou réglementaires sont nombreuses lorsque l'on a affaire aux administrations complexes des grands États modernes : différents et parfois nombreux (particulièrement en France) ministères, directions multiples au sein de ceux-ci, agences ou établissements administratifs divers... Chacune de ces entités peut se comporter de facto comme une organisation ou une quasi organisation dont les objectifs propres sont plus ou moins bien reliés aux objectifs des politiques gouvernementales. Le problème de la cohérence de l'action gouvernementale est en permanence posé par la différenciation des organes de l'administration nécessaires à l'exercice de missions extrêmement variées mais source possible de contradictions, de redondances et de complexité des législations et réglementations. La fonction d'intégration au profit du chef de l'exécutif, Président ou Premier Ministre, est d'autant plus nécessaire. Le processus de l'AIR joue un rôle en la matière. Aux États-Unis c'est l'OIRA (office of impact régulation assesment) sous ensemble de l'Office of budget and management (OMB) dépendant directement de la Maison Blanche qui joue ce rôle au profit de la présidence. En France c'est le secrétariat général du gouvernement (SGG). L'AIR peut jouer un rôle effectif dans cette fonction de contrôle au profit du responsable de l'exécutif dès lors que les modes d'élaboration des analyses sont édictés par ces entités, ce qui est le cas dans les deux pays cités et qu'elles reçoivent ces études dans le cadre du processus d'élaboration des textes avant l'adoption de ceux-ci et sont donc en mesure de réagir à leur contenu. On remarquera que si ce paradigme est assez clairement revendiqué aux États-Unis, il l'est de façon beaucoup plus discrète en France ou de manière générale le SGG est rarement mis en avant dans le débat public ${ }^{19}$ sans que l'importance de son rôle soit mise en doute par les initiés.

Les neuf paradigmes que l'on vient de passer rapidement en revue ainsi que celui, beaucoup plus vague, de bonne législation, nous renvoient à trois types d'ordre (au sens d'espèce ou de catégorie Cf. Littré) : l'ordre juridique, l'ordre démocratique et l'ordre économique : le désir d'une bonne législation, dans sa version de légistique formelle, la volonté de lutter contre les lois bavardes, ainsi que le désir de centralisation, sinon de la fabrique du moins du contrôle des textes relèvent d'abord de l'ordre juridique dès lors qu'on inclut dans les valeurs de celui-ci la normativité du droit, la hiérarchie des normes, leur absence de contradictions... La mise en cause du pouvoir discrétionnaire, l'équilibrage des pouvoirs, la référence à une démocratie participative, la redevabilité des gouvernants ainsi que la boucle évaluative - dans la version où elle serait d'abord un outil de redevabilité -relèvent de l'ordre démocratique par l'incarnation de certaines conceptions de la démocratie. L'invocation du libéralisme économique, de la simplification administrative, de la légistique matérielle, ainsi que de la boucle évaluative dans son aspect d'outil de pilotage relèvent d'abord de l'ordre économique. ${ }^{20}$

Ce rattachement principal d'un paradigme -ou de l'une de ses versions- à un ordre n'exclut pas un rattachement second à l'un ou l'autre des deux autres ordres. D'autant que

\footnotetext{
19 Jean Maïa « la légistique au secrétariat général du gouvernement « in CJFI 2008 p 21-25

${ }^{20}$ Le rattachement d'un paradigme à un ordre a été effectué en fonction de notre interprétation de la finalité du paradigme, il ne préjuge en rien des modes d'opérationnalisation de ce paradigme. Ainsi la démocratie participative est classée dans l'ordre démocratique car elle vise pour ses tenants à un approfondissement de la démocratie. Ceci posé la démocratie participative requiert pour s'exercer des normes juridiques, mais ce besoin se situe au niveau des moyens d'exercice, non de la finalité du paradigme.
} 
les paradigmes entre eux, ne sont pas mutuellement exclusifs et que les frontières entre les ordres sont poreuses Ces rattachements seconds sont matérialisés dans le tableau synoptique ci-dessous par un x là où les rattachements principaux le sont par xxx.

Tableau 1 : Croisement entre paradigmes et ordres

\begin{tabular}{|c|c|c|c|}
\hline & Ordre juridique & Ordre démocratique & Ordre économique \\
\hline $\begin{array}{l}\text { Mise en cause } \\
\text { du pouvoir } \\
\text { discrétionnaire }\end{array}$ & $x$ & $x x x$ & $x$ \\
\hline $\begin{array}{l}\text { Équilibrage } \\
\text { des pouvoirs }\end{array}$ & & $x x x$ & \\
\hline $\begin{array}{l}\text { Démocratie } \\
\text { participative }\end{array}$ & & $x x x$ & $x$ \\
\hline $\begin{array}{l}\text { Libéralisme } \\
\text { économique }\end{array}$ & & $x$ & $x x x$ \\
\hline $\begin{array}{l}\text { Simplification } \\
\text { administrative }\end{array}$ & $x$ & $x$ & $x x x$ \\
\hline $\begin{array}{l}\text { Lutte contre } \\
\text { les lois bavardes }\end{array}$ & $x x x$ & & \\
\hline Bonne législation & $x x x F 1$ & & $x x x F 2$ \\
\hline $\begin{array}{l}\text { Redevabilité } \\
\text { des gouvernants }\end{array}$ & & $x x x$ & \\
\hline Boucle évaluative & & $\mathrm{xxx} F 2$ & $x x x F 1$ \\
\hline $\begin{array}{l}\text { Centralisation de la } \\
\text { fabrique des textes }\end{array}$ & $x x x$ & & \\
\hline
\end{tabular}

\section{Caractéristiques de l'appropriation de I'AIR en France}

La France en adoptant l'étude d'impact des projets de lois s'est inscrite dans le mouvement de l'AIR porté par l'Organisation de coopération et de développement économique, mais cette adoption s'est naturellement caractérisée par une appropriation particulière que l'on va maintenant s'efforcer de caractériser.

Pour cela nous ne partirons pas d'une estimation des impacts (Outcome) qu'a pu produire l'AIR. Ceux-ci ont pu, de manière générale être identifiés, pour toute évaluation, comme pouvant relever de quatre types (Johnson, 1998) : l'utilisation instrumentale qui désigne l'utilisation des résultats d'une évaluation comme une base de l'action, l'utilisation conceptuelle qui a trait « au changement conceptuel dans la façon dont les acteurs inclus dans son processus perçoivent la problématique de la politique (policy issue) » (ibidem), l'utilisation politique qui vise à conforter la position des acteurs qui l'adoptent à l'égard de la politique concernée, et l'utilisation processuelle 
qui a trait à l'apprentissage que procure l'évaluation à ses parties prenantes quant à la capacité de mieux maîtriser le raisonnement évaluatif. L'utilisation de cette grille est intéressante et à même de différencier de façon significative des AIR concrètes ${ }^{21}$ mais elle requiert une méthodologie fondée sur des études de cas en profondeur, différente de celle que nous avons utilisée pour le présent article ainsi, du moins peut-on en faire l'hypothèse, qu'un minimum de maturité dans les appropriations qui ne paraît pas actuellement atteinte en France.

Pour notre part nous nous contenterons de mettre en lumière une appropriation, essentiellement manifeste, appréhendée au niveau des réalisations (outputs). Nous distinguerons, de façon un peu schématique, le genre et l'intensité de cette appropriation. L'identification du genre sera faite par les choix effectués entre les paradigmes dominants ainsi que leur priorisation dans l'expérience française (Choix et priorisation pouvant être conscients ou inconscients, explicites ou latents). L'estimation de l'intensité sera effectuée à partir de l'observation du comportement des acteurs dans la « chaîne » de production-contrôle de l'étude d'impact.

\subsection{Caractérisation à partir de la domination de certains paradigmes}

Les doxas développées au niveau international ou dans chaque État se gardent bien d'insister sur les coûts d'opportunités qui peuvent exister lorsque l'on donne la priorité à certains paradigmes plutôt qu'à d'autres du fait des antagonismes que l'on peut relever entre les divers paradigmes. On a plutôt le sentiment d'une sorte de convergence des intérêts qu'il y aurait à s'inspirer de ces divers paradigmes dont le respect traduirait la prédominance du bon sur le mauvais, du souhaitable sur le regrettable. La réalité est bien évidemment plus complexe et les rapports entre paradigmes peuvent aller d'une forte proximité à un antagonisme avéré en passant par une ambivalence assez forte et une indifférence presque totale.

C'est ainsi que le paradigme de rééquilibrage des pouvoirs paraît indifférent à celui de la lutte contre le pouvoir discrétionnaire, l'usage comme l'abus de ce dernier pouvant être la propension tant du pouvoir exécutif que du pouvoir législatif. Ce paradigme de rééquilibrage paraît également indifférent à la démocratie participative, celle-ci se situant plutôt en amont des prises de décision de l'un ou (et) de l'autre des deux pouvoirs. En revanche le paradigme de rééquilibrage des pouvoirs paraît antagoniste avec la simplification administrative en raison de la propension des parlementaires à multiplier les amendements de toute nature aux projets de loi, source de complexité quand ils sont adoptés, avec le libéralisme (même raison), avec la lutte contre les lois bavardes (idem), avec la bonne législation (du fait du caractère souvent improvisé des amendements et de leur finalité souvent clientéliste), avec la redevabilité des gouvernants, avec la boucle évaluative ainsi qu'avec la centralisation de la fabrique des textes.

Pour sa part le paradigme de la démocratie participative est indifférent au paradigme libéral, la participation pouvant pousser à l'interventionnisme ou au libéralisme selon la nature des revendications, préoccupations et intérêts (claims, concerns and interests) des parties prenantes (Lincoln et Guba, 1989) et leurs rapports de force. Ce paradigme est antagoniste avec ceux de la simplification administrative -en raison de la complexité et de la variété des intérêts en présence et de la difficulté d'assurer compromis et équilibres dans les satisfactions qu'on leur accorde-, de la lutte contre les lois bavardes dans la mesure où une façon de tenter de surmonter les divergences des «claims, concerns and interests » des parties prenantes est de se réfugier

${ }^{21}$ Pour un exemple sur ce point cf. Rissi et Sager 2013. 
dans des objectifs de nature très générale, dans l'abstraction, ou d'adopter des « postures de la vertu » (Gibert et Verrier, 2016) peu contraignantes et susceptibles, par là même, de recueillir une large adhésion. Le paradigme de la démocratie participative paraît également antagoniste avec le paradigme de la centralisation et du contrôle de la fabrique des textes dans la mesure où chacun de ces textes peut être marqué par un équilibre délicat résultant de la démarche participative dont rien ne permet d'espérer la compatibilité avec d'autres textes provenant d'autres démarches participatives caractérisées par la prépondérance d'autres intérêts et d'autres rapports de force. La démocratie participative entretient avec la bonne législation des rapports qui peuvent être considérés comme ambivalents puisqu'elle peut assurer une meilleure prise en compte des besoins, problèmes et cadres cognitifs des parties prenantes mais dans le même temps cet apport positif peut être obéré par une incapacité à se dégager de positions antagonistes, ainsi que par un rallongement important du processus de confection des textes. Le paradigme de la démocratie participative entretient aussi une ambivalence dans ses rapports avec la redevabilité des gouvernants du fait de la dilution de responsabilité qu'engendre la co-construction de textes normatifs, de même qu'avec la boucle évaluative dont l'élément premier - clarté, priorisation et précision des objectifs -peut faire à l'occasion cruellement défaut (Cf. supra).

En continuant à faire des hypothèses sur les relations de compatibilité entre paradigmes nous avons bâti une matrice qui se présente ainsi :

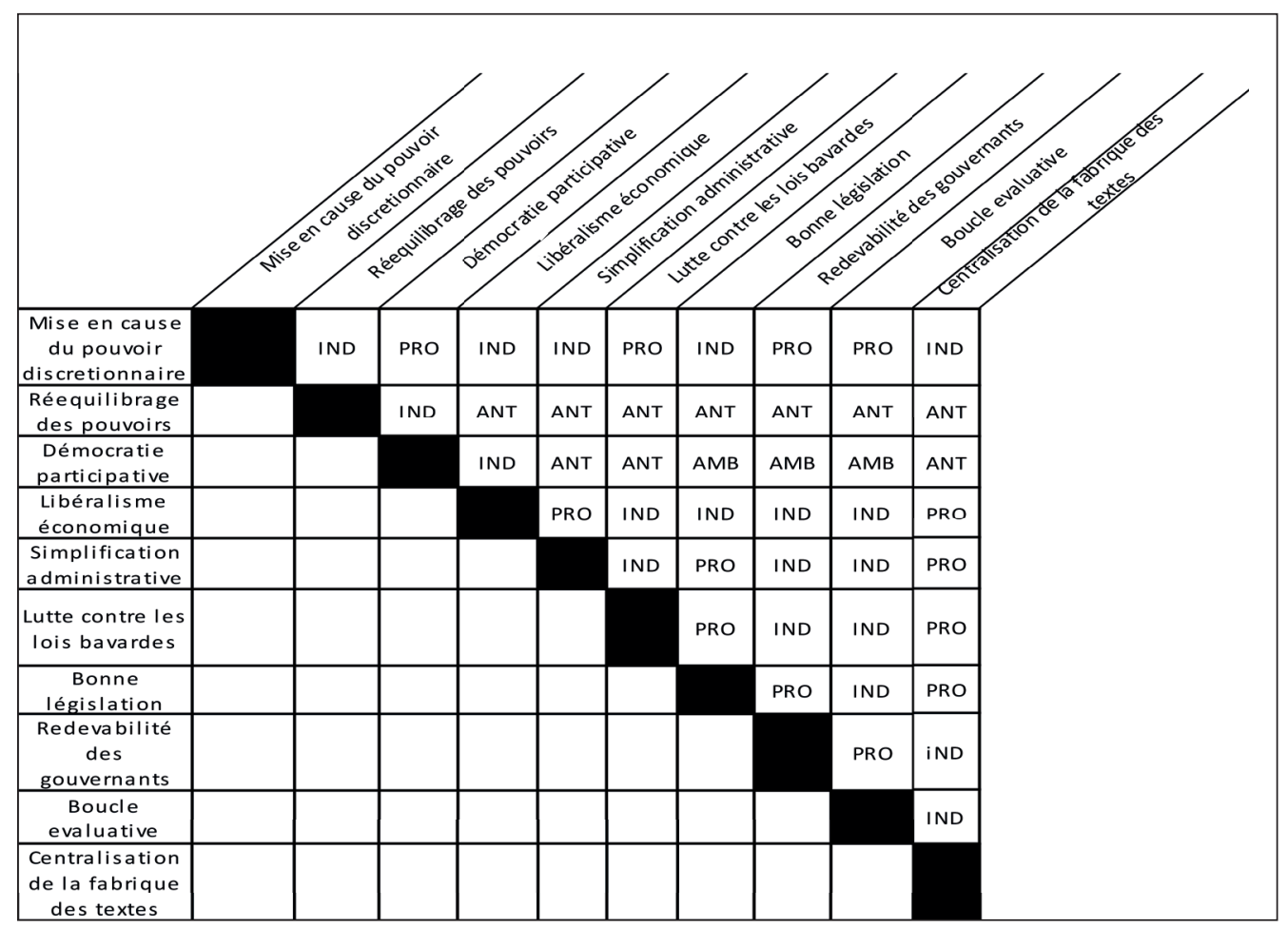

Nota : IND pour rapport d'indifférence. AMB pour ambivalence des rapports. ANT pour rapports antagonistes. PRO pour rapports positifs. 
Jusqu'ici la situation française comparée à ce qui est rapporté des expériences étrangères se caractérise par une relative euphémisation du libéralisme économique et de la mise en cause du pouvoir discrétionnaire qui lui est proche, on ne saurait par exemple y voir un terrain d'application du Cost Benefit State (Sunstein, 1996) tel que l'on a pu le dénoncer dans le cas des États-Unis, un recours modéré à la notion de bonne législation à laquelle est préférée l'expression plus scientifique et moins moralisatrice de légistique (Biland et Vanneuville, 2012; Philippart, 2011)

De la même façon redevabilité des gouvernants, boucle évaluative, et démocratie participative sont évoquées, dans les débats et les documents administratifs mais sans apparaitre comme des finalités majeures. À l'inverse la simplification administrative apparaît, dans les discours du moins, comme une compagne de route pérenne de l'étude d'impact. Le rapport de la commission d'information de l'assemblée nationale sur la simplification législative donne une bonne idée de la proximité dans les milieux politiques français des notions de simplification d'une part et d'étude d'impact d'autre part : les 2/3 du rapport sont consacrés à l'étude d'impact!

Le paradigme de rééquilibrage des pouvoirs -sans doute à cause du déséquilibre initial établi par la constitution de la $\mathrm{V}^{\mathrm{e}}$ république- est également très présent dans le débat, la loi organique qui a instauré l'étude d'impact incluait également d'autres mesures visant explicitement ce rééquilibrage. La lutte contre les lois bavardes partage cette caractéristique même si elle est essentiellement portée par les juges administratifs et constitutionnels. L'évocation de la nécessité d'améliorer la confection des lois est omniprésente, enfin la centralisation de la fabrique des textes apparaît comme un paradigme fort de conséquences concrètes alors même qu'il n'est évoqué que de façon discrète.

Si l'on ne retient que les cinq paradigmes auxquels il est fait un fort recours et que l'on s'attache à la nature des relations que l'on a estimé être les leurs (Cf. schéma ci-dessous), l'on note qu'il reste cinq relations de proximité, quatre relations antagonistes et l'une d'indifférence.

\begin{tabular}{|l|l|l|l|l|}
\hline $\begin{array}{c}\text { Mise en cause } \\
\text { du pouroir } \\
\text { discretionnaire }\end{array}$ \\
\hline $\begin{array}{c}\text { Réequilibrage } \\
\text { des pouvoirs }\end{array}$ \\
\hline $\begin{array}{c}\text { Démocratie } \\
\text { participative }\end{array}$
\end{tabular}


Ce qui révèle encore des contradictions dans la façon dont les parties prenantes françaises se sont approprié l'étude d'impact, contradictions dues à la seule référence au paradigme de rééquilibrage des pouvoirs. Le fait que les propositions de lois ainsi que les amendements des parlementaires aussi bien que gouvernementaux soient dispensés d'étude d'impact soulève évidemment des problèmes au regard des autres objectifs qui tous quatre peuvent être rangés sous la vaste et peu définie ombrelle de rationalisation de l'action publique, le problème a d'ailleurs été bien vu (Cf. rapport AN 2014) sans qui lui soit jusqu'à présent porté remède.

\subsection{Caractérisation à partir du comportement des parties prenantes}

L'appropriation de l'analyse d'impact de la réglementation peut être étudiée à partir des comportements de chacune des parties prenantes d'une étude. Celles-ci sont constituées par les assujettis à l'étude à savoir le ou les ministères porteur(s) du projet, les auteurs de l'étude, l'auditeur de l'étude lorsqu'existe une procédure de contrôle de la qualité de celle-ci -, dans, le cas de l'étude d'impact législatif il s'agit du Conseil d'État- des destinataires de l'étude, prioritairement les parlementaires et le cas échéant les citoyens - et parmi eux les parties prenantes à la politique considérée-(dans la mesure où les études sont publiées et peuvent faire l'objet d'observations) et finalement le juge, en l'occurrence le juge constitutionnel puisqu'il peut censurer un texte législatif à raison des insuffisances d'une étude.

Chacun de ces groupes d'acteurs peut bien évidemment manifester une appropriation différente de celle des autres groupes quant aux paradigmes qu'il valorise, l'intensité de l'intérêt qu'il accorde à l'instrument, la vision de cet instrument comme une contrainte, une ressource ou un objet ambivalent... mais on peut avancer l'hypothèse d'une interdépendance des appropriations de ces différents acteurs : les exigences des destinataires, du juge et de l'auditeur rejaillissant normalement sur l'interprétation par les auteurs et les assujettis aux études (les ministères porteurs des projets) ainsi que par les auteurs de celles-ci, très généralement des services internes à ces ministères de la nature, de la profondeur, de la rigueur du travail à effectuer ainsi que sur l'observation de l'ensemble des dispositions de la loi et de ses textes d'application.

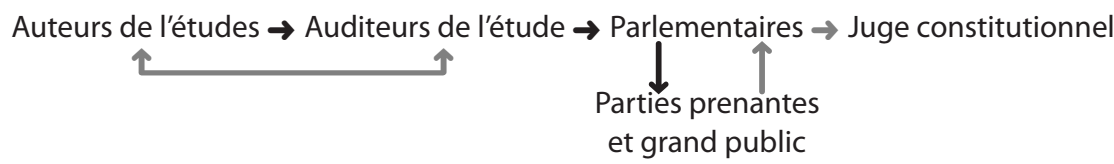

On traitera d'abord du travail des assujettis et des auteurs des études, la différence étant difficile à faire dans la mesure où très généralement les auteurs appartiennent à des services internes $\mathrm{au}(\mathrm{x})$ ministère(s) concernés(s). Pour cela nous avons effectué une analyse des études d'impact des cinq premiers projets de loi soumis au parlement par le gouvernement dirigé par Édouard Philippe : le projet de loi «renforçant la sécurité intérieure et la lutte contre le terrorisme » $\left(\mathrm{SILT}^{22}\right)$, le projet de loi organique « rétablissant la confiance dans l'action publique » (CAP).

\footnotetext{
${ }^{22}$ Les acronymes utilisés, sont de l'auteur, sont présentés par pure commodité pour la lecture texte et ne sont pas consacrés.
} 
Le projet de loi d'habilitation à prendre par ordonnances les mesures pour le renforcement du dialogue social (RDS), la loi de programmation des finances publiques pour les années 2018 à 2022 (LPFP), le projet de loi « mettant fin à la recherche ainsi qu'à l'exploitation des hydrocarbures conventionnels et non conventionnels et portant diverses dispositions relatives à l'énergie et à l'environnement (hydro). Cet ensemble de projets soumis au pouvoir législatif en début de mandat présidentiel témoigne de la variété des types de texte pour lesquels les études d'impact sont réalisées, non seulement quant aux domaines concernés : sécurité et libertés publiques, statut (au sens le plus large du terme) des gouvernants, travail, finances publiques, énergie et développement durable mais aussi quant aux types de loi : organique (CAP), ordinaires (SILT et Hydro), de finances (LPFP) et d'habilitation (RDS). Et cela en dépit des dispenses ou allégements d'études prévus par l'article 11 de la loi organique de $2009^{23}$.

Chaque étude d'impact, conformément à une recommandation du guide de légistique, mis sur pied par le secrétariat général du gouvernement se compose d'une introduction plus ou moins brève ainsi que de développements consacrés à chacun des points demandés par la loi organique (cf. encadré $\mathrm{n}^{\circ} 1$ ) par article ou groupes d'articles. Ainsi le document intitulé étude d'impact est-il une succession d'études (que l'on dénommera élémentaires pour éviter la confusion) dont chacune reprend les différents items de la loi organique mais apparaît comme indépendantes des autres. Dans le panel examiné le nombre d'études élémentaires a varié de 5 (CAP) à 11 (SILT).

Le travail de caractérisation a été effectué pour chaque étude élémentaire, les résultats indiqués pour une étude d'impact de projet étant la sommation des résultats des études élémentaires qu'elle contient (et le cas échéant des caractérisations de la partie introductive commune placée avant les études élémentaires).

L'approche choisie a été purement quantitative, pour éviter des redondances par rapport aux analyses qualitatives déjà effectuées sur un certain nombre d'études et pour tester la possibilité d'une « objectivisation » du type d'appropriation.

L'indicateur utilisé pour caractériser les développements consacrés à chaque item prescrit par la loi organique a été le nombre de signes (espaces exclus). La mise en œuvre de cet indicateur a rencontré d'importants problèmes dus à la nécessité de reclasser des développements, souvent présentés dans des rubriques auxquelles manifestement ils ne ressortissaient pas. L'existence de ces problèmes et des interprétations nécessitées par leurs solutions fait que la précision des chiffres est quelque peu illusoire et que seuls les ordres de grandeur doivent être pris en compte.

Les principaux résultats de l'étude sont présentés dans le tableau 2 infra. Les options possibles en dehors de l'intervention de règles de droit nouvelles, tiennent toujours une très faible place dans les études d'impact. Elles ne font jamais l'objet d'un examen approfondi. À l'intérieur de l'agrégat option internes à la législation nouvelle ou présentation de la nouvelle législation -que nous avons dû constituer en raison de la très forte interpénétration des deux types de développements dans les études élémentaires- les parts relatives du détail de la présentation

\footnotetext{
${ }^{23}$ « l'article 8 n'est pas applicable aux projets de révision constitutionnelle, aux projets de loi de finances, aux projets de loi de financement de la sécurité sociale, aux projets de loi de programmation visés au vingt et unième alinéa de l'article 34 de la Constitution ainsi qu'aux projets de loi prorogeant des états de crise. Les dispositions des projets de loi par lesquelles le Gouvernement demande au Parlement, en application de l'article 38 de la Constitution, l'autorisation de prendre des mesures par ordonnances sont accompagnées, dès leur transmission au Conseil d'État, des documents visés aux deuxième à septième alinéas et à l'avant-dernier alinéa de l'article 8 ».
} 


\begin{tabular}{|c|c|c|c|c|c|c|c|c|c|c|c|c|}
\hline$\stackrel{\text { จे }}{\mathrm{O}}$ & iे & $\stackrel{\circ}{\stackrel{\circ}{\sigma}}$ & ৯े & ণे & $\stackrel{\circ}{\circ}$ & $\stackrel{\circ}{\stackrel{\circ}{\sigma}}$ & $\stackrel{\circ}{\circ}$ & $\stackrel{\circ}{\circ}$ & ळे & ৯े & $\stackrel{\circ}{\circ}$ & ठ̊ํ \\
\hline $\begin{array}{l}\text { ठे } \\
\text { ठ }\end{array}$ & 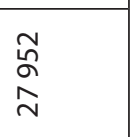 & $\begin{array}{l}\text { n } \\
\text { N } \\
\text { D }\end{array}$ & $\begin{array}{l}\infty \\
\infty \\
\stackrel{\infty}{\simeq}\end{array}$ & 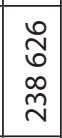 & $\begin{array}{c}0 \\
\infty \\
\infty \\
n\end{array}$ & $\begin{array}{l}\hat{0} \\
\circ \\
\curvearrowright\end{array}$ & $\begin{array}{l}\text { Һ̆ } \\
\text { m }\end{array}$ & $\begin{array}{l}\underset{\sim}{\infty} \\
\underset{0}{0}\end{array}$ & $\begin{array}{l}m \\
\stackrel{0}{0} \\
\infty \\
\simeq\end{array}$ & $\begin{array}{l}0 \\
m \\
m \\
=\end{array}$ & $\underset{N}{\stackrel{N}{N}}$ & \begin{tabular}{|l} 
\\
$\frac{0}{0}$ \\
m \\
in
\end{tabular} \\
\hline ठ̊ & $\stackrel{\circ}{\circ}$ & $\stackrel{\text { \} }{\check{1}}} &{\text { ஓे }} &{\text { \े}} &{\text { ठ̊ }} &{\stackrel{\stackrel{\circ}{\mathrm{m}}}{\mathrm{O}}} &{\stackrel{\circ}{\circ}} &{\stackrel{\circ}{\circ}} &{\text { 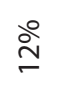 }} &{\text { ळ }} &{\text { ठे }} &{\text { ঃ̊ }} \\
{\hline \stackrel{\widehat{m}}{\stackrel{\gamma}{\sigma}}} &{\stackrel{\mathscr{Y}}{\mathscr{Y}}} &{\underset{\infty}{\stackrel{n}{n}}} &{\text { 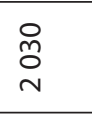 }} &{\begin{array}{l} \\
\text { ò } \\
\text { in } \\
\end{array}} &{ } &{\text { 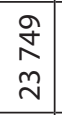 }} &{\stackrel{\infty}{\wedge}} &{\tilde{N}} &{\underset{\substack{\infty \\
\infty}}{\stackrel{m}{N}}} &{\begin{array}{l}\text { n } \\
\text { in } \\
\text { in }\end{array}} &{ } &{\begin{array}{l}0 \\
\frac{9}{\delta} \\
\frac{1}{1}\end{array}} \\
{\hline \stackrel{\circ}{\grave{N}}} &{\text { ळे }} &{\text { 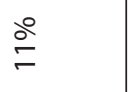 }} &{\stackrel{\text { mे }}{\circ}} &{\stackrel{\circ}{\stackrel{0}{\sim}}} &{\text { ○े }} &{\stackrel{8}{\circ}} &{\text { ○े }} &{\text { ठ̊ }} &{\text { in }} &{\text { ஓे }} &{\text { ல̊ }} &{\text { ఫे }} \\
{\hline \begin{array}{l}\text { مू } \\
\stackrel{o}{\sigma} \\
\stackrel{\sim}{n}\end{array}} &{\text { 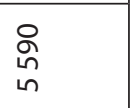 }} &{\frac{n}{\infty}} &{\underset{\sim}{\stackrel{q}{N}}} &{\begin{array}{|l}0 \\
0 \\
0 \\
0 \\
\end{array}} &{ } &{\begin{array}{l}n \\
\tilde{\sigma} \\
=\end{array}} &{ } &{\text { in }} &{\begin{array}{l}m \\
\bar{n} \\
m\end{array}} &{\underset{\sim}{\stackrel{N}{N}}} &{\stackrel{\infty}{\stackrel{\infty}{\sigma}}} &{\begin{array}{l}\tilde{\tilde{O}} \\
\stackrel{0}{n}\end{array}} \\
{\hline \text { }} &{ } &{ } &{\stackrel{\circ}{\circ}} &{\text { ลे }} &{\stackrel{\circ}{\circ}} &{\stackrel{े}{\circ}} &{ } &{ } &{ } &{ } &{ } &{\text { ๙े }} \\
{\hline \begin{array}{l}\text { 命 } \\
\text { m} \\
m\end{array}} &{ } &{ } &{\text { 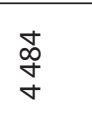 }} &{\begin{array}{l}8 \\
\text { o } \\
\text { o } \\
\end{array}} &{\begin{array}{l} \\
\stackrel{2}{N} \\
- \\
\end{array}} &{\begin{array}{l}\text { aे } \\
\infty \\
\text { in }\end{array}} &{ } &{ } &{ } &{ } &{ } &{\text { 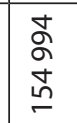 }} \\
{\hline \text { 욤 }} &{\text { ウे }} &{\stackrel{\circ}{\stackrel{\circ}{\circ}}} &{\text { ঃे }} &{\text { 商 }} &{\text { in }} &{\stackrel{\infty}{\circ}} &{\text { ஓे }} &{\text { ஓ̊ }} &{\text { ウे }} &{\text { ठ̊ }} &{\text { ঃे }} &{\text { ஃे }} \\
{\hline \begin{array}{l}\text { o } \\
\stackrel{\infty}{+} \\
m \\
m\end{array}} &{\text { 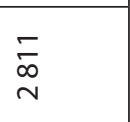 }} &{\begin{array}{l}\hat{N} \\
\hat{n} \\
\sim\end{array}} &{ } &{\begin{array}{l}\hat{\kappa} \\
\tilde{o} \\
\text { on }\end{array}} &{\begin{array}{l}\bar{\sigma} \\
\sigma\end{array}} &{\begin{array}{l}\stackrel{\sim}{\sigma} \\
\sim\end{array}} &{\stackrel{\infty}{\stackrel{\infty}{\sim}}} &{\begin{array}{l}\infty \\
\kappa \\
\text { ñ }\end{array}} &{\stackrel{\stackrel{\sim n}{q}}{\sim}} &{ } &{ } &{\begin{array}{l}\text { ¿্ } \\
\text { \&े }\end{array}} \\
{\hline \stackrel{\circ}{\wedge}} &{\stackrel{\circ}{\circ}} &{\stackrel{\circ}{\grave{N}}} &{\stackrel{\sim}{\circ}} &{\text { o̊ }} &{\text { 8̊ }} &{\text { 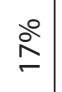 }} &{\text { ठ̊ }} &{\stackrel{\circ}{\circ}} &{\text { 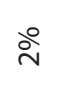 }} &{\text { ৯े }} &{\text { 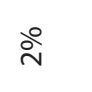 }} &{\text { ठे }} \\
{\hline \text { 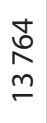 }} &{\begin{array}{l}\stackrel{\infty}{\sim} \\
\stackrel{\sigma}{\leftarrow}\end{array}} &{\begin{array}{l}\stackrel{8}{ } \\
\infty \\
\hat{m}\end{array}} &{\underset{\sim}{\stackrel{\forall}{\sim}}} &{\begin{array}{|l|}8 \\
0 \\
0 \\
\circ \\
0\end{array}} &{ } &{\begin{array}{l}a \\
\frac{1}{0} \\
0 \\
0\end{array}} &{ } &{\text { 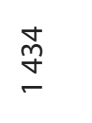 }} &{\underset{m}{\stackrel{N}{m}}} &{\text { 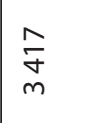 }} &{\begin{array}{l}\hat{n} \\
\hat{n} \\
m\end{array}} &{\text { 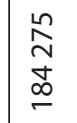 }} \\
{\hline \text { 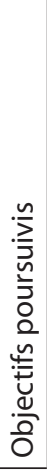 }} &{\text { 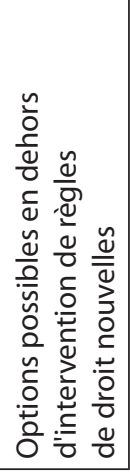 }} &{\text { 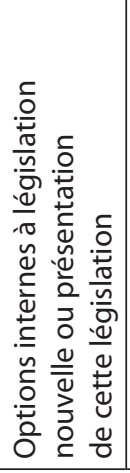 }} &{\text { 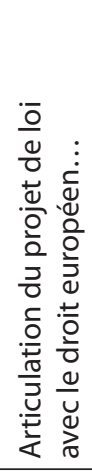 }} &{\text { 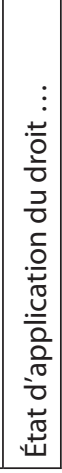 }} &{\text { 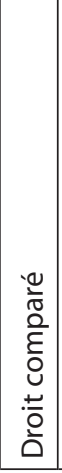 }} &{\text { 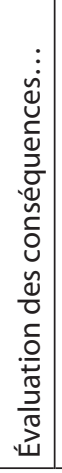 }} &{\text { 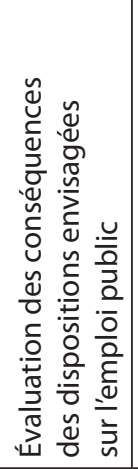 }} &{\text { 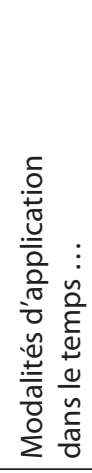 }} &{\text { 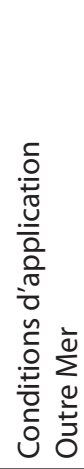 }} &{\text { 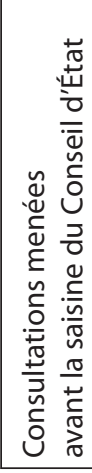 }} &{\text { 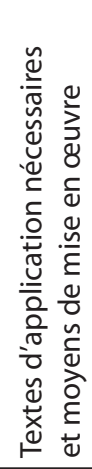 }} &{\begin{array}{l}\overline{0} \\
\stackrel{0}{0}\end{array}} \\
$\hline
\end{tabular}


et des options internes sont très variables, ces dernières sont fréquemment absentes mais sont parfois détaillées de façon assez complète. Les développements consacrés à l'état d'application du droit tiennent toujours une place importante au sein des études, Cette place est largement prépondérante dans l'étude d'impact de RDS ou un véritable cours de droit régnant du travail et de ses inconvénients est donné. L'évaluation des conséquences de toute nature (cf. l'encadré) poste qui correspond le mieux à l'appellation générique d'étude d'impact occupe généralement une place faible, l'étude sur hydro faisant exception. ${ }^{24}$ Ces impacts ne sont généralement pas quantifiés (l'étude sur l'hydro faisant là aussi exception), leur méthode d'évaluation n'est donc pas fournie; qui plus est dans la chaîne de production publique allant des moyens aux impacts en passant par les activités ou les réalisations, ils se situent très rarement au niveau des effets au regard des objectifs affichés mais plutôt au niveau des réalisations. Quand on note aussi que parmi les conséquences figurent des conséquences juridiques, on constate qu'il y a un jeu qu'illustre bien le passage du terme d'étude d'impact à celui d'évaluation des conséquences qui devient un terme ombrelle pour des développements fourre-tout.

Dans le tableau 3 on a repris l'idée des trois ordres auxquels on a rattaché les différents paradigmes invoqués comme finalités des études d'impact et tenté de classer chacun des items susmentionnés comme outils apportant à l'un des trois ordres. La transversalité de deux items (objectifs et Outre-Mer) par rapport à au moins de deux des trois ordres nous ayant semblé forte nous les avons affectés à une catégorie supplémentaire.

Ce tableau suggère, dans quatre des cinq cas, la très forte prégnance de l'ordre juridique, la faiblesse de l'ordre démocratique (qui aurait pu apparaître moindre si on avait rangé parmi ces outils les options externes au lieu de les ranger dans l'ordre économique, mais qui serait demeurée réelle compte tenu de la rareté des développements consacrés à ces options externes), et la modestie de l'ordre économique. Sur cet échantillon la place consacrée aux thèmes juridiques, l'emporte de très loin sur tout ce qui pourrait ressembler à des développements de calcul économique.

Compte tenu des limites des validités interne et externe de ces résultats l'étude de l'appropriation en France de l'étude d'impact doit être complétée par un essai de synthèse de constats faits par ailleurs ou par d'autres.

Les exigences du juge se révèlent faibles (Hutier, 2015; Gibert et Verrier, 2016). Trois possibilités lui étaient offertes de censurer un texte pour insuffisance de l'étude d'impact ayant accompagné son dépôt au Parlement : sur la base de l'article 61-1 de la constitution et du mécanisme de la question préalable de constitutionnalité pour une loi déjà promulguée, sur la base de l'article 61 avant promulgation de la loi et sur celle de l'article 39 avant inscription d'un projet de loi à l'ordre du jour de l'assemblée la première saisie par le gouvernement ${ }^{25}$. Le Conseil

${ }^{24}$ Qui plus est les pourcentages calculés surestiment généralement les développements réels : les intitulés des items prenant parfois autant sinon plus de place que les développements qu'ils recouvrent de type par exemple « sans objet».

Pour RDS il est seulement indiqué que les impacts feront l'objet de fiche à partir des ordonnances, ce qui semble logique dans la mesure où ce sont celles-ci qui contiendront les mesures juridiques détaillées mais qui aboutit à un paradoxe certain dans la mesure où les lois de ratification des ordonnances n'ont pas à faire l'objet d'études d'impact. En d'autres termes l'habilitation est demandée au nom d'objectifs généraux sans qu'il y ait dans le process loi d'habilitation- ordonnances- loi de ratification des ordonnances une explicitation des effets concrets attendu des mesures prises en vertu de l'habilitation, sans parler de leur quantification.

${ }^{25}$ Extrait de l'article 39 « Les projets de loi ne peuvent être inscrits à l'ordre du jour si la Conférence des présidents de la première assemblée saisie constate que les règles fixées par la loi organique sont méconnues. En cas de désaccord entre la Conférence des présidents et le Gouvernement, le président de l'assemblée inté- 
Tableau 3 : Estimation de la part des développements relevant de chacun des trois ordres

\begin{tabular}{|c|c|c|c|c|c|c|c|}
\hline & ITEMS INCLUS & SILT & CAP & RDS & LFPP & Hydro & Moyenne \\
\hline $\begin{array}{l}\text { Ordre } \\
\text { juridique }\end{array}$ & $\begin{array}{l}\text { État d'application du } \\
\text { droit.. Options internes } \\
\text { à la législation nouvelle } \\
\text { ou présentation de cette } \\
\text { législation... Articulation } \\
\text { du projet de loi avec le } \\
\text { droit européen... Droit } \\
\text { comparé... Modalités } \\
\text { d'application dans } \\
\text { le temps... Textes } \\
\text { d'application }\end{array}$ & $62 \%$ & $67 \%$ & $74 \%$ & $47 \%$ & $39 \%$ & $58 \%$ \\
\hline $\begin{array}{l}\text { Ordre } \\
\text { démocratique }\end{array}$ & Consultations & $2 \%$ & $0 \%$ & $0 \%$ & $3 \%$ & $8 \%$ & $3 \%$ \\
\hline $\begin{array}{l}\text { Ordre } \\
\text { économique }\end{array}$ & $\begin{array}{l}\text { Options possibles } \\
\text { en dehors d'intervention } \\
\text { de règles de droit } \\
\text { nouvelles. Évaluation } \\
\text { des conséquences... } \\
\text { Emploi public }\end{array}$ & $27 \%$ & $15 \%$ & $4 \%$ & $24 \%$ & $35 \%$ & $21 \%$ \\
\hline Commun & $\begin{array}{l}\text { Objectifs poursuivis... } \\
\text { Application Outre Mer }\end{array}$ & $9 \%$ & $18 \%$ & $22 \%$ & $26 \%$ & $18 \%$ & $19 \%$ \\
\hline Total & & $100 \%$ & $100 \%$ & $100 \%$ & $100 \%$ & $100 \%$ & $100 \%$ \\
\hline
\end{tabular}

Constitutionnel a d'emblée exclu l'idée d'utiliser l'article 61-1. Il annonçait dès son examen de la loi organique instaurant les modalités de mise en œuvre de l'article 39 de la constitution ${ }^{26}$, au moyen de deux réserves interprétatives ${ }^{27}$, qu'il apprécierait de façon souple le respect des dispositions de cette loi concernant l'étude d'impact. Il a conditionné son acceptation de se prononcer sur l'inconstitutionnalité qu'entraînerait une malfaçon d'étude d'impact au fait que l'invocation de cette malfaçon ait été faite en conférence des présidents, même si la conférence n'a pas donné de suites à cette invocation, se comportant alors de facto comme un juge d'appel (Hutier, 2015). Enfin on notera que sur la quinzaine de fois où il a été saisi dans le cadre de l'article 61 d'une demande de censure d'une loi votée, au titre d'une malfaçon d'étude d'impact le juge n'a jamais prononcé cette censure ou celle de certaines des dispositions de la loi, alors que les insuffisances des études au regard des dispositions de l'article 8 étaient flagrantes pour beaucoup d'observateurs.

ressée ou le Premier ministre peut saisir le Conseil constitutionnel qui statue dans un délai de huit jours. » ${ }^{26}$ Décision n²009-579 DC du 9 avril 2009.

${ }^{27}$ La première énonçait que l'exigence de procéder à une étude correspondant à chacune des rubriques énumérées par cet article ne s'imposait que pour celles de ces rubriques qui apparaissaient pertinentes au regard de l'objet de la loi. La seconde indiquait que "dans le cas où un projet de loi serait déposé sans être accompagné d'une étude d'impact satisfaisant en totalité ou en partie aux prescriptions de l'article 8, il apprécierait le respect de ces dispositions au regard des exigences de la continuité de la vie de la Nation ». 
L'auditeur des projets de lois est le Conseil d'État agissant en tant que conseiller du gouvernement (et non en tant que juridiction administrative). Tous les projets de lois lui sont présentés pour avis et c'est dans le cadre de l'examen qu'il effectue pour étayer et rendre cet avis qu'il est amené à exprimer son appréciation sur la qualité des études d'impact. Qualifier l'appropriation de l'AIR qui est la sienne est délicat du fait que les avis du conseil d'État ont longtemps été non publics, l'avis étant donné au seul gouvernement qui est maître de leur publication ou non ${ }^{28}$. Si l'on s'en tient aux lois ordinaires (en excluant traités et accords ainsi que ratification d'ordonnances) le taux de publication des avis est important pour les textes accompagnés d'étude d'impact $55 \%$ et adoptés par l'assemblée nationale de janvier 2016 à mars 2017 mais on ne peut exclure un biais important de non-publication. Dans ses rapports comme dans les auditions de ses dirigeants, le Conseil d'État se présente à la fois comme partisan voire initiateur de l'étude d'impact- préconisée dans son rapport annuel de 2006-, désireux que soient supprimées les voies de contournement de celles-ci et assez critique de la qualité des études d'impact réalisées jusqu'ici. L'examen de ses avis publiés (Cf. annexe) ne révèle pas toujours une sévérité bien grande sur des études d'impact qui peuvent apparaître à l'observateur comme lacunaires ou superficielles, le terme de satisfaisant décerné à ces études ne semble pas révéler une investigation profonde. En 2014 le Vice-président du Conseil d'État déclarait «En tout et pour tout, nous n'avons jusqu'à présent rejeté qu'un seul texte pour absence ou quasi-absence d'étude d'impact » (colloque AN, 2014) Le Conseil d'ailleurs met volontiers en avant la faiblesse du temps que lui laisse le gouvernement pour rendre ses avis comme un élément de difficulté majeure de son travail.

Quant au Parlement, s'il proteste volontiers quand il estime que l'Europe ne fait pas suffisamment la démonstration de la nécessité des contraintes qu'elle veut imposer aux États membres ${ }^{29}$, il ne fait guère montre aux dires mêmes des parlementaires du même appétit de démonstration quand il s'agit d'examiner les projets de loi d'initiative nationale (AN, 2014).

\section{Une appropriation qui est bien loin de confirmer la montée effective de la rationa- lité économique et managériale dans l'élaboration des règles et des politiques}

On ne peut qu'être frappé par l'isomorphisme coercitif que révèle la loi organique ${ }^{30}$. Un même type d'analyse avec les mêmes composantes est requis pour tous les projets de loi quels que soient les modes d'action publique utilisés dans ces projets, les contextes dans lesquels ils s'insèrent, le caractère plus ou moins instrumental ou symbolique des politiques qu'ils portent, l'homogénéité de la politique qu'ils promeuvent ou à l'inverse l'hétérogénéité des dispositifs qu'ils intègrent. C'est la nature juridique du texte (projet de loi) qui détermine les obligations à respecter pour justifier le texte à l'exclusion de toute autre considération. On

\footnotetext{
${ }^{28}$ Même si fréquemment des fuites transparaissaient dans la presse.

${ }^{29} \mathrm{Cf}$ par exemple la Résolution Européenne AN 933 de l'Assemblée nationale.

${ }^{30}$ Même si le guide de légistique, pour sa part introduit l'idée d'une certaine contingence : « Le format de l'étude et le degré d'approfondissement de l'analyse peuvent être assez variables selon les projets de loi, le principe étant qu'ils soient proportionnés à l'importance de la réforme envisagée, à la difficulté de la matière et à l'importance des impacts qui en résulteront pour les citoyens ou les entreprises, y compris s'ils ne concernent qu'une petite partie d'entre eux, mais avec de lourds enjeux. De même, l'approche devra être adaptée en fonction de la nature du texte et des marges de manœuvre juridiques disponibles »
} 
trouve là un phénomène très semblable à celui que nous avons pu noter à propos des Projets annuels de performance (PAP) et des rapports annuels de performance (RAP) en appui des demandes budgétaires inscrites dans le projet la loi de finances pour ceux-là, du reporting en appui du projet de la loi de règlement pour ceux-ci (Benzerafa et Gibert, 2016). On ne constate pas l'équivalent dans la plupart des pratiques étrangères ou les textes devant être l'objet d'une AIR sont déterminés le plus souvent au cas par cas (Union Européenne par exemple), ce qui semble indiquer pour le moins l'existence d'un doute quant à la pertinence ou l'utilité de l'AIR pour tout type de texte. Il peut paraître quelque peu paradoxal de voir que c'est le juge constitutionnel qui dans ses réserves interprétatives émises lorsque la loi organique de 2009 lui a été soumise avant promulgation ${ }^{31}$ a fait œuvre de contextualisation en énonçant que l'exigence de procéder à une étude correspondant à chacune des rubriques énumérées par la loi organique ne s'imposait que pour celles de ces rubriques qui apparaissaient pertinentes au regard de l'objet de la loi, mais aussi que «dans le cas où un projet de loi serait déposé sans être accompagné d'une étude d'impact satisfaisant en totalité ou en partie aux prescriptions de l'article 8, il apprécierait le respect de ces dispositions au regard des exigences de la continuité de la vie de la Nation».

En matière d'étude d'impact des projets de lois, la situation française se caractérise donc par un contraste entre une forte institutionnalisation, un champ très large des objets soumis à étude ainsi que des items à inclure dans cette étude et une faible appropriation par le système constitué par l'ensemble des parties prenantes et leurs interactions. Ce système inclut un juge qui laisse pour essentiel la responsabilité de l'acceptation ou non d'une étude au pouvoir législatif et un pouvoir législatif (colloque AN, 2014) dont les membres n'attachent que peu d'importance aux dites études sauf pour nourrir leur rapport ou y voir un outil parmi d'autres pour s'opposer aux desiderata du pouvoir exécutif lorsqu'ils sont dans l'opposition -utilisation opportuniste-. Il intègre aussi un auditeur (le Conseil d'État) qui milite pour un renforcement des études et de leur champ, mais est prompt à dégager sa responsabilité dans la légèreté du contrôle des études en mettant l'accent sur les seuls délais qui lui sont laissés et jamais sur les problèmes d'expertise que les exigences de la méthode soulèveraient en son sein. Il inclut des auteurs des études qui sont placés sous l'autorité hiérarchique des porteurs des projets qu'ils sont censés évaluer et que rien n'incite (cf. ce qui précède) à prendre des risques en renforçant l'objectivité et la rigueur de leur analyse. Une telle situation peut avoir deux grands types d'explications non exclusives l'une de l'autre.

La première résiderait dans la nature même de l'outil dont la doxa prise au pied de la lettre tend à limiter le champ des possibles pour les gouvernants et à les obliger à mettre en valeur des solutions dont ils ne veulent pas, des effets sur lesquels ils préfèrent garder le silence... en d'autres termes à adopter une posture de la vertu que les modes, l'air du temps, obligent à prendre mais qui se traduirait par une simple cotisation symbolique à une rationalité qui n'est pas forcément la leur. En outre l'AIR est par nature techniquement délicate. Les problèmes d'appropriation de l'AIR peuvent tenir à la nature de l'objet évalué; il s'agit d'un texte juridique, et non d'un programme qui se caractérise généralement par des objectifs relativement précis ou une politique qui dans son sens le plus large recouvre l'ensemble des interventions de la puissance publique dans un domaine déterminé (culture, défense...). L'objet retenu présente divers risques d'hétérogénéité quant aux objectifs

${ }^{31}$ Décision n²009-579 DC du 9 avril 2009. 
poursuivis, aux parties prenantes, aux hypothétiques chaînes causales de référence ${ }^{32} \ldots$ L'une d'entre elle est celle, soulignée, par Bussmann (2010) entre la visée d'ordre social et la visée de résolution de problèmes, la première est centrée sur des normes touchant au comportement des personnes ou des organisations, la seconde est centrée sur l'atteinte d'un résultat désiré. Le mélange des deux visées dans un même texte est des plus communs, mais les logiques et les faisabilités d'évaluation diffèrent fortement (ibidem)

Seule les études systématiques de la réalité de l'AIR dans les pays étrangers peuvent permettre d'étayer cette hypothèse d'un modèle idéal type de l'étude d'impact plus mythique que réaliste.

La seconde explication est plus culturaliste, elle est centrée sur la façon dont la France met en œuvre l'ensemble des réformes qui visent à une rationalisation de l'action publique, manière que l'on pourrait traduire par un «faire semblant» largement développé. Cette explication a été avancée par le Vice-Président du Conseil d'État qui a qualifié l'administration française de championne dans l'édification de «villages Potemkine » quand il s'agit de réformes (Conseil d'État, 2016). De façon plus centrée sur le problème de l'étude d'impact une autre explication culturelle est avancée : En France on s'attacherait surtout « tant à clarté formelle de la norme qu'à son caractère lisible et compréhensible par le plus grand nombre ». À l'étranger «l'accent est davantage mis sur les effets de la norme sur l'individu ou l'activité économique et sur la charge administrative qu'elle représente. » (Idem) Il est évident que si l'on admet cette hypothèse le problème d'une plus profonde appropriation de l'Analyse d'Impact de la réglementation relève du changement cognitif beaucoup plus que de la mise en œuvre de nouvelles procédures destinées à élargir le champ des études -aux amendements gouvernementaux par exemple ainsi qu'aux ordonnances- ou à renforcer l'audit de celles-ci. C'est pourtant vers l'instauration de ces procédures que se fixent les vœux ou les recommandations de réforme, tant de certains parlementaires (AN, 2014) que du Conseil d'État (Conseil d'État, 2016). Il y a donc un paradoxe relativement inquiétant pour les tenants de l'AIR.

On peut voir une force de la prégnance culturelle dans le contraste que l'on peut observer en France entre l'usage de la légistique formelle et celui de la légistique matérielle

La disposition de la loi organique de 2009 concernant l'insertion des projets de lois ou d'accord internationaux examinés dans le droit existant européen ou national, l'obligation faite aux auteurs des études d'impact d'énoncer la liste des textes d'application qui seront nécessaires à la mise en œuvre du texte de loi soumis au parlement (Cf. encart $\left.n^{\circ} 1\right)$, le respect effectif de ces obligations dans les études d'impact réalisées, de même que le passage obligé par le secrétariat général du gouvernement ou encore l'exercice de l'audit des études par le Conseil d'État montrent que dans l'appropriation de l'étude d'impact le souci de la cohérence juridique apparaît comme fondamental; un texte doit trouver sa place dans l'ordre juridique existant quel que soit le degré de novation qu'il contient. Cette cohérence juridique, qui concerne à la fois le respect de la hiérarchie des normes et la non-contradiction entre des normes de niveau équivalent est sans doute nécessaire à l'État de droit et à sa crédibilité, elle est cependant très différente de la cohérence des politiques publiques. Il est vrai qu'avec celle-ci elle partage une chose : un texte ne saurait être apprécié uniquement en lui-même. Il doit être replacé dans un ensemble, ou portefeuille, de textes juridiques d'une part, de politiques publiques d'autre part

32 En théorie la réalisation d'études élémentaires article par article ou groupe d'articles (cf. supra) par groupes d'articles est une réponse à ce problème de l'hétérogénéité 
si on veut correctement évaluer ex ante ce qu'il est susceptible d'apporter aux ordres juridique et social existants. La cohérence juridique ne garantit pas la cohérence de l'action publique. On peut avoir des projets de lois favorisant par exemple l'activité des entreprises par des aides ou dégrèvements divers et en même temps des textes qui rendent difficile cette activité sans que ces textes ne présentent de contradiction juridique les uns avec les autres. Qui plus est le contrôle de la cohérence juridique est sans doute globalement plus simple que le contrôle de la cohérence de l'action car nécessitant moins d'interprétations. L'accent mis sur la cohérence juridique n'est donc pas un gage de rationalisation de l'action de l'État si par rationalisation on entend cohérence de l'action (ceci étant il n'est pas toujours évident de faire la part entre incohérence et fine tuning). La légistique formelle l'emporte donc sur la légistique matérielle (Martens et Morand, 2000; Dellet et Flueckiger, 2005), les études ne font pratiquement jamais appel à la méthode coût-avantage qui dans la doxa en est pourtant un instrument central. Le faible recours à la légistique matérielle suggère qu'au binôme de deux légistiques se substitue un binôme légistique formelle et dialectique éristique. Cette dernière, art de la controverse, qui culmine avec l'art d'avoir toujours raison (Schopenhauer), est renouvelée par les emprunts de forme qu'elle effectue à la rationalité économico-manageriale se coulant ainsi, à peu de frais, dans le moule d'une modernité apparente. par la grâce d'un accord plus ou moins implicite entre les acteurs les plus importants du système d'élaboration- audit - utilisation - contrôle juridictionnel de l'étude d'impact.

\section{Bibliographie}

Aggeri F. et Labatut J., 2010. La gestion au prisme de ses instruments. Une analyse généalogique des approches théoriques fondées sur les instruments de gestion. Finance Contrôle Stratégie 13 (3), 5-37.

Alemanno A., 2015. Dans quelle mesure le « Mieux légiférer » est-il meilleur? Une première analyse de l'impact de la nouvelle initiative « Mieux légiférer » sur 1〉Union européenne. Revue du Droit de l'Union Européenne 4, 509.

Arndt C., 2008. The Politics of Governance Ratings. International Public Management Journal 11 (3), 275-297.

Auby J.-B. et Perroud T. (Eds), 2013. Regulatory Impact Assessment. Global Law Press, Seville. Bartoli A., Jeannot J. et Larat F., 2016. La simplification des formes et modalités de l'action publique : origines, enjeux et actualité. Revue française d'administration publique 157, 7-22.

Benzerafa-Alilat M. et Gibert P., 2016. De quoi l'État rend-il compte dans ses rapports annuels de performance? Revue française d'administration publique 160, 1 041-1 064.

Biland E. and Vanneuville R., 2012. Government Lawyers and the Training of Senior Civil Servants. Maintaining Law at the Heart of the French State. International Journal of the Legal profession 19 (1), 29-54,

Bussmann W., 2010. Evaluation of Legislation: Skating on thin Ice. Evaluation 16 (3), 279-293.

Chevallier J., 2016. La simplification de l'action publique et la question du droit. Revue française d'administration publique $157,205-214$.

Chmielowski J. and Waldemar H., 2015. Regulatory Impact Assessment (RIA) and Rationality of Law-Legal Aspects. Management and Business Administration 23 (2), 92-108.

Chun Y. H. and Rainey H., G. 2005. Goal Ambiguity in US Federal Agencies. Journal of Public Administration Research and Theory 15 (1), 1-30.

Combrade B.-L., 2014. L'étude d'impact au Parlement français : un instrument de mutation du rôle des assemblées dans le processus législatif? Revue française d'administration publique 149, 195-206.

Costa Mia, Desmarais B. A. and Hird J. A., 2016. Science Use in Regulatory Impact Analysis: The Effects of Political Attention and Controversy. Review of Policy Research, 33 (3), 251-269. 
Dellet J.-D. et Flueckiger A., 2005. La légistique : une élaboration méthodique de la législation In Drago J., Confection de la loi, Presses Universitaire de France, Paris, 83-96

Denolle A.-S., 2011. Les études d'impact : une révision manquée? Revue française de droit constitutionnel 87 (3), 499-514.

Desmarais B. A. and Hird J. A., 2014. Public Policy's Bibliography: The Use of Research in US Regulatory Impact Analyses. Regulation \& Governance 8 (4), 497-510.

Devriendt É. et Monte M., L'exposé des motifs : un discours d'autorité. Le cas des lois françaises de 2003, 2010 et 2014 sur les retraites. Mots. Les langages du politique [En ligne], 107 I 2015,

Dunlop C. A., Maggetti M., Radaelli C. M. et al., 2012. The many Uses of Regulatory Impact Assessment: A Meta-Analysis of EU and UK cases. Regulation \& Governance 26 (1), 23-45.

Dunlop . A. and Radaelli C. M. (ed.)., 2016. Handbook of Regulatory Impact Assessment. Edward Elgar Publishing, London.

Elder C. D. and Cobb R. W., 1984. Agenda-Building and the Politics of Aging. Policy Studies Journal 13 (1), 115.

Flûckiger A., 2007. L'évaluation législative ou comment mesurer l'efficacité des lois. Revue européenne des sciences sociales XLV (138), 83-101.

Francesco F. De., 2012. Diffusion of regulatory impact analysis among OECD and EU Member States. Comparative Political Studies 45 (10), 1277-1305.

Gibert P., 2002. L'analyse de politique à la rescousse du management public? Ou la nécessaire hybridation de deux approches que tout, sauf l'essentiel. Politiques et management public 20 (1), 1-14.

Gibert P. et Pierre-Éric V., 2016. Peut-on discipliner le pouvoir ? Étude sur le contrôle de la rationalité managériale par le juge dans trois innovations législatives françaises. Politiques et management public 33 (3-4), 165-196.

Halpern C., Lascoumes P. et Le Galès P. (dirs), 2014. L'instrumentation de l'action publique. Les presses de Sciences Po, Paris.

Hahn R. W., Burnett T., Jason K., Chan Yee-Ho I. 2000. Assessing Regulatory Impact Analysis: The failure of Agencies to Comply with Executive Order 12,866. Harvard Journal of Law and Public policy 23 (3), 859.

Hahn. W. and Dudley P. M., 2007. How Well Does the US Government Do Benefit-Cost Analysis? Review of Environmental Economics and Policy 1 (2), 192-211.

Hood C., 1986. The Tools of government. Springer, New York

Hutier S., 2015. Retour sur un moyen récurrent : les malfaçons de l'étude d'impact des projets de loi. Revue française de droit constitutionnel 101,73-86.

Issalys P., 2013. Analyse d'impact et production normative de l'efficacité à la légitimité. Revista da Faculdade de direito da UFMG, 45-274.

Jacob S., Speer S., Furubo J.-E., 2015. The Institutionalization of Evaluation Matters: Updating the International Atlas of Evaluation 10 years later. Evaluation 21 (1), 6-31.

Johnson R. B., 1998 Toward a theoretical model of evaluation utilization. Evaluation and Program Planning $21(1), 93-110$

KPMG et Quadrant Conseil, 2017. Évaluation de la démarche globale d'évaluation des politiques publiques menée dans le cadre de la modernisation de l'action .

Lamarque D., 2016. Contrôle et évaluation de la gestion publique. Enjeux contemporains et comparaisons internationales. Bruylant. Bruxelles.

Lincoln Y. S., Guba E. G., 1989. Fourth generation evaluation. Sage, London.

Löfstedt R. E., 2004. The Swing of the Regulatory Pendulum in Europe: From Precautionary Principle to (Regulatory) Impact Analysis. Journal of Risk and Uncertainty 28 (3), 237-260.

March J. G. and Olsen J., 1989. Rediscovering institutions. The organizational basis of politics . The Free Press, New York.

Martens P., 2000. Morand Charles-Albert (sous la dir.), Légistique formelle et matérielle. Formal and Material Legistic. Droit et société 46, 731-733. 
Moor M., 1995. Creating Public Value. Strategic management in Government . Harvard University Press. Harvard.

Muller P., 2005. .Esquisse d'une théorie du changement dans l'action publique. Structures, acteurs et cadres cognitifs. Revue française de science politique 55, 155-187.

Oliver C., 1991. Strategic Responses to Institutional Processes. The Academy of Management Review 16 (1), 145-179.

Philippart P., 2011. Au confluent du droit et de la gestion : la légistique pour évaluer l'utilisation de la dimension entrepreneuriale de la loi sur l'Innovation. Management \& Avenir 50 (10), 15-37

Radaelli C. M., 2004. The Diffusion of Regulatory Impact Analysis: Best Practice or Lesson $\square$ Drawing? European Journal of Political Research 43 (5), 723-747.

Radaelli C. M., 2005. Diffusion without Convergence: how Political Context Shapes the Adoption of Regulatory Impact Assessment. Journal of European Public Policy 12 (5), 924-943.

Radaelli C. M. and De Francesco F., 2010. Regulatory Impact Assessment In Baldwin R., The oxford handbook of regulation. Oxford University Press, Oxford, 279-301

Rissi C. and Sager F., 2013. Types of Knowledge Utilization of Regulatory Impact Assessments: Evidence from Swiss policymaking. Regulation \& Governance 7 (3), 348-364.

Rose-Ackerman S., 2010. Putting Cost-Benefit Analysis in Its Place: Rethinking Regulatory Review. U. Miami L. Rev. 65,335 .

Shapiro S. and Morrall J., 2016. Does Haste Make Waste? How Long Does It Take to Do a Good Regulatory Impact Analysis? Administration \& Society 48 (3) 367-389.

Speer S , Pattyn V. and De Peuter B., 2015. The Growing Role of Evaluation In Parliaments: Holding Governments Accountable? International Review of Administrative Sciences, 81 (1) 37-57.

Sunstein C. R., 2015. The Cost-Benefit State. International Review of Administrative Sciences 81 (1), 37-57.

Star S. L., 2010. Ceci n'est pas un objet-frontière! Revue d'anthropologie des connaissances 4 (1), 18-35.

Star S. L. and Griesemer J. R., 1989. Institutional Ecology, 'Translations' and Boundary Objects: Amateurs and Professionals in Berkeley's Museum of Vertebrate Zoology, 1907-39. Social Studies of Science 19 (3), 387-420.

Troper M. 2011. Argumentation et explication. Droits 54 (2), 3-26.

De Vaujany F. X., 2006. Pour une théorie de l'appropriation des outils de gestion : vers un dépassement de l'opposition conception-usage. Management \& Avenir 9, 109-126.

\section{Documents administratifs et assimilés}

\section{France :}

Assemblée Nationale, 2014. Rapport d'information fait au nom de la mission d'information sur la simplification administrative.

Autorité environnementale, 2017. Note de l'Autorité environnementale sur les évaluations socio-économiques des projets d'infrastructures linéaires de transport Note délibérée $\mathrm{n}^{\circ}$ 2017-N-05.

Charpin J.-M., Ruat L et Freppel C., 2016. Évaluation des procédures d'évaluation socio-économique des projets d'investissements publics. Inspection générale des finances, Paris .

Colloque « Mieux légiférer» (colloque AN 2014) Assemblée nationale Vendredi 28 novembre 2014. Compte rendu http://www.assemblee-nationale.fr/presidence/colloque-mieux-legiferer-28-novembre-2014.pdf

Commission indépendante : L'impact de l'Accord Économique et Commercial Global entre l'Union européenne et le Canada (AECG/CETA) sur l'environnement, le climat et la santé Rapport au Premier ministre. Septembre 2017.

Conseil d'État, 2016. Étude annuelle 2016 Simplification et qualité du droit. 
Conseil national d'évaluation des normes, 2016. Rapport public d'activité 2015 . Août 2016.

Conseil Constitutionnel, 2009. Décision no 2009-579 DC du 9 avril 2009 NOR : CSCX0908392S loi organique relative à l'application des articles $34 * 1,39$ et 44 de la constitution. 16 avril 2009 Journal officiel de la république française.

Goasguen C. et Mallot J., 2009. Rapport d'information fait au nom du comité d'évaluation et de contrôle des politiques publique sur les critères de contrôle des études d'impact accompagnant les projets de loi, $\mathrm{N}^{\circ} 2094$ Assemblée nationale Novembre 2009.

La légistique ou l'art de rédiger le droit (CJFI 2008). Numéro Spécial Courrier juridique des finances et de l'industrie juin 2008.

Premier Ministre, 2013. Circulaire du 17 juillet 2013 relative à la mise en œuvre du gel de la réglementation.

Premier ministre, 2015. Évaluation des normes et qualité du droit.

Premier Ministre, 2016. Circulaire relative à l'Evaluation de l'impact sur la jeunesse des projets de loi et de textes réglementaires.

Sauvé J.-M., 2010. Vice-président du Conseil d'État Le rôle du Conseil d'État dans la mise en œuvre des études d'impact Conférence organisée par l'Université Paris Ouest Nanterre La Défense Lundi 29 novembre 2010 Cercle France Amériques.

Etudes d'impact : ensemble des études relatives au texte de lois « adoptés » par l'assemblé nationale sur les quinze mois janvier 2016- avril 2017.

\section{États-Unis}

Clinton W. J., 1993. Executive Order 12866 of September 30, 1993 Regulatory Planning and Review Federal Register Presidential Documents Vol. 58, No. 190 Monday, October 4, 1993.

Nixon R. Executive order 12291 federal regulation. https:/www.archives.gov/federal-register/codification/ executive-order/12291.html

Obama B., 2011. Executive Order 13563 -- Improving Regulation and Regulatory Review. 18 janvier 2011

Office of management and budget (OMB 2003). Circular A-4 September 17, 2003 To the Heads of Executive Agencies and Establishments Subject: Regulatory Analysis Improving regulatory impact analysis through process reform.

Trump D., 2017. Presidential Executive Order on Reducing Regulation and Controlling Regulatory Costs. January 30, 2017.

\section{$O C D E$}

Organisation de Coopération et de Développement Économiques (OCDE 2008) Introductory Handbook for Undertaking Regulatory Impact Analysis (RIA) Version 1.0 October 2008.

Deighton-Smith R., Erbacci A. and Kauffmann C., 2016. Promoting inclusive growth through better regulation: The role of regulatory impact assessment. OECD Regulatory Policy Working Papers, No. 3, OECD Publishing, Paris. http://dx.doi.org/10.1787/5jm3tqwqp1vj-en

OCDE. Recommandation du conseil concernant la politique et la gouvernance réglementaires.

\section{Union Européenne}

European Commission. Guidelines on Impact Assessment. http://ec.europa.eu/smartregulation/guidelines/ug_ chap3_en.htm

Commission européenne, 2009. Lignes directrices concernant l'étude d'impact de la réglementation 15 janvier 2009.

Cour des comptes européenne, 2010. L'analyse d'impact dans les institutions européennes soutient-elle la prise de décision? Rapport spécial n³ 2010.

Parlement européen, 2014. Résolution du 27 novembre 2014 sur la révision des lignes directrices de la Commission concernant l'analyse d'impact et le rôle du test PME (2014/2967(RSP). 


\section{Annexe 1 : L'échantillon d'études analysé}

(Dénomination des projets de loi et des articles ou groupe d'articles faisant l'objet d'études élémentaires dans le cadre de l'étude d'impact du projet de loi)

\section{Projet de loi renforçant la sécurité intérieure et la lutte contre le terrorisme}

1 : Périmètres de protection

2 : Fermeture des lieux de culte aux fins de prévenir des actes de terrorisme.

3 : Mesures individuelles de surveillance des personnes présentant une menace d'une particulière gravité pour la sécurité et l'ordre publics aux fins de prévenir des actes de terrorisme

4 : Visites et saisies

5 et 6 : Transposition de la directive 2016/681 PNR

7 : Mise en œuvre d'un traitement automatisé de données à caractère personnel pour le transport maritime de passagers

8 et 9 : La surveillance des communications hertziennes

10 : Contrôle dans les zones frontalières

11 : Conditions d'application outremer

\section{Projet de loi organique rétablissant la confiance dans l'action publique}

Titre 1 : dispositions relatives au Président de la République - Article 1

Titre II : dispositions relatives aux parlementaires - Chapitre premier- dispositions relatives aux conditions d'éligibilité et inéligibilités - Article 2

Chapitre II : dispositions relatives aux incompatibilités - Articles 3 à 8

Chapitre III : dispositions supprimant « la réserve parlementaire - Article 9

Titre III : dispositions relatives au médiateur du crédit aux candidats et aux partis politiques - Article 10

\section{Projet de loi d'habilitation à prendre par ordonnances les mesures pour le renfor- cement du dialogue social}

Article $1^{\text {er }}$ : dispositions relatives à l'articulation des normes et à la négociation collective Article 2 : dispositions renforçant le dialogue social

Article 3 : dispositions relatives à la sécurisation des relations de travail

Article 4 : dispositions favorisant le développement de la négociation collective et sécurisant les accords collectifs

Article 5 : dispositions modifiant les obligations des employeurs en matière de pénibilité et de détachement de travailleurs

Article 7 : ajustements relatifs a la législation en matière de travail du dimanche

Article 9 : décalage d'un an de l'entrée en vigueur du prélèvement a la source de l'impôt sur le revenu 


\section{Loi de programmation des finances publiques pour les années 2018 à 2022}

Article 19 : interdiction du recours au crédit-bail immobilier pour l'état et ses opérateurs Article 20 : recensement des garanties octroyées au cours de l'année

Article 21 : autorisation du recours à l'emprunt pour le fonds de garantie et de résolution des dépôts

Article 22 : remise d'un rapport sur la situation financière des établissements publics de santé Article 23 : rénovation des relations financières entre l'État et la sécurité sociale

Article 24 : nouvelle règle prudentielle de maîtrise de la dépense et de l'endettement local Article 26 : rapport annexé au projet de loi de finances, relatif au grand plan d'investissement Article 27 : bilan de la mise en œuvre de la LPFP

Article 28 : rapport annexé au plf relatif aux opérateurs « jaune opérateurs » Article 29 : abrogation de la LPFP 2014-2019

\section{Projet de loi mettant fin à la recherche ainsi qu'à l'exploitation des hydrocarbures conventionnels et non conventionnels et portant diverses dispositions relatives à l'énergie et à l'environnement.}

Chapitre I : arrêt de l'exploration et de l'exploitation des hydrocarbures, chapitre VI dispositions relatives à l'exploitation outremer - Articles 1, 2, 3 et 8

Chapitre II : dispositions relatives au stockage et aux consommateurs de gaz - Article 4 Chapitre III : dispositions relatives aux relations entre fournisseurs et gestionnaires de réseau - Article 5

Chapitre IV : dispositions relatives aux contrôles des biocarburants - Article 6

Chapitre $\mathbf{V}$ : dispositions relatives à la réduction des émissions de certains polluants atmosphériques - Article 7 